\title{
ABSTRACT EVOLUTION EQUATIONS AND THE MIXED PROBLEM FOR SYMMETRIC HYPERBOLIC SYSTEMS
}

\author{
BY \\ FRANK J. MASSEY III( $\left.{ }^{1}\right)$
}

\begin{abstract}
In this paper we show that Kato's theory of linear evolution equations may be applied to the mixed problem for first order symmetric hyperbolic systems of partial differential equations.
\end{abstract}

1. Introduction. This paper is concerned with the mixed problem for the following symmetric hyperbolic system of partial differential equations:

$$
\begin{aligned}
\frac{\partial u}{\partial t}+\sum_{j=1}^{m} a_{j}(x, t) \frac{\partial u}{\partial x_{j}}+b(x, t) u & =f(x, t), & & x \in \Omega, \quad 0 \leqq t \leqq T ; \\
u(x, 0) & =\phi(x), & & x \in \Omega ; \\
u(x, t) & \in P(x, t), & & x \in \Gamma, \quad 0 \leqq t \leqq T .
\end{aligned}
$$

The unknown $u=\left(u_{1}, \ldots, u_{N}\right)$ is a real vector-valued function, the coefficients, $a_{j}$ and $b$, are real $N \times N$ matrix-valued functions, and the $a_{j}$ are symmetric. We assume $a_{j}$ and $b$ are of class $C^{2}$ and $C^{1}$ on $\bar{\Omega} \times[0, T]$, respectively. $\Omega$ is a bounded open subset of $R^{m}$ with boundary $\Gamma$ of class $C^{3}$.

The results are restricted to the regular case where the boundary matrix

$$
a_{n}(x, t)=\sum n_{j}(x) a_{j}(x, t), \quad x \in \Gamma, \quad 0 \leqq t \leqq T,
$$

is nonsingular on $\Gamma \times[0, T]$. Here $n=\left(n_{1}, \ldots, n_{m}\right)$ is the exterior unit normal to $\Omega$. Limits of summation will be omitted when they are clear from the context.

The boundary subspace $P(x, t)$ is a linear subspace of $R^{N}$ which varies in a $C^{3}$ manner with $(x, t) \in \Gamma \times[0, T]$, and it is maximal nonnegative for each $x, t$. This means

$$
\left(a_{n}(x, t) u, u\right) \geqq 0, \quad u \in P(x, t),
$$

and $P(x, t)$ is not contained in any other subspace of $R^{N}$ having this property. Here $(u, v)$ denotes the usual inner product of $u, v \in R^{N}$.

Received by the editors May 25, 1971.

AMS 1970 subject classifications. Primary 35L50; Secondary 34G05, 35F15, 35L40, 35L45, 35R20, 47A50, 47D05.

Key words and phrases. Symmetric hyperbolic systems, evolution equations, mixed problem, $C_{0}$-semigroups, Cauchy problem.

(1) This research was supported by Air Force Office of Scientific Research grant AFAFOSR-68-1462 and National Science Foundation grant NSF GP-8257.

Copyright (C) 1972, American Mathematical Society 
Symmetric hyperbolic systems have been studied by Friedrichs ([4], [5]), Lax and Phillips [10], Cordes and Moyer [2], and others. Here we treat equation (1.1) using Kato's results [9] on linear evolution equations of the form

$$
d u / d t+A(t) u=f(t), \quad 0 \leqq t \leqq T,
$$

where the linear operators $-A(t)$ are the infinitesimal generators of $C_{0}$-semigroups in a Banach space. Kato established sufficient conditions for the existence of the evolution operator $\{U(t, s): 0 \leqq s \leqq t \leqq T\}$ for the family $\{A(t)\}$, and he applied these results to the equation (1.1) in the case where $\Omega=R^{m}$ is the whole space. In this paper we extend Kato's method to the case where $\Omega$ is a bounded domain.

Before proceeding further, we make some remarks concerning notation. We shall reserve the terminology vector-valued function for a function $u=\left(u_{1}, \ldots, u_{N}\right)$ which has $N$ real-valued components. $X$ and $L^{2}(\Omega)$ will both be used to denote the Hilbert space of square-integrable vector-valued functions on $\Omega . H^{k}(\Omega), k=0,1$, $\ldots$, is the Sobolev space of those $u \in X$ whose partial derivatives of order up to $k$ also lie in $X . H^{-k}(\Omega)$ is the dual space of $H_{0}^{k}(\Omega)$, where $H_{0}^{k}(\Omega)$ is the closure in $H^{k}(\Omega)$ of the set of $C^{\infty}$ vector-valued functions with compact support in $\Omega$. The spaces $L^{2}\left(R^{m}\right), H^{k}\left(R^{m}\right), H^{-k}\left(R^{m}\right)$ are the corresponding spaces on $R^{m} . L^{2}(\Gamma)$ is the space of vector-valued functions on $\Gamma$ which are square integrable with respect to the natural surface measure on $\Gamma$. The Sobolev space $H^{k}(\Gamma), 0 \leqq k \leqq 3$, consists of those vector-valued functions on $\Gamma$ which coincide on coordinate neighborhoods with functions in $H^{k}\left(R^{m-1}\right)$, and $H^{-k}(\Gamma)$ is the dual space to $H^{k}(\Gamma)$. Unless otherwise stated, Sobolev spaces $H^{k}$ are of integral order $k$, so $0 \leqq k \leqq 3$, for example, means $k=0,1,2,3$. We use \|\| and $($,$) to denote the norm and inner product$ in $X, L^{2}\left(R^{m}\right)$, or $L^{2}(\Gamma)$, and \|\|$_{k}$ to denote the norm in $H^{k}(\Omega), H^{k}\left(R^{m}\right)$, or $H^{k}(\Gamma)$. The underlying space, $\Omega, R^{m}$, or $\Gamma$, should be clear from the context.

If $u \in H^{k}(\Omega), 1 \leqq k \leqq 3$, then $u_{0} \in H^{k-1}(\Gamma)$ denotes the trace of $u$ on $\Gamma$. If $P(x)$, $x \in \Gamma$, is a linear subspace of $R^{N}$ which varies continuously with $x$, then $H_{P}^{1}(\Omega)$ denotes the closed subspace of $H^{1}(\Omega)$ consisting of those $u$ which satisfy the boundary conditions $u_{0}(x) \in P(x)$ for (almost all) $x \in \Gamma$. For basic properties of Sobolev spaces, see Hörmander [6], Lions and Magenes [11], Morrey [12], and Seeley [13].

If $u \in R^{N}$, then $|u|$ denotes the usual Euclidean norm of $u$. If $a$ is an $N \times N$ matrix, then $|a|=\sup \{|a u|:|u|=1\}$, and ${ }^{t} a$ is the transpose of $a$.

If $X_{1}, X_{2}$ are Banach spaces, then $B\left(X_{1}, X_{2}\right)$ denotes the space of bounded operators from $X_{1}$ to $X_{2}$, and $B\left(X_{1}\right) \equiv B\left(X_{1}, X_{1}\right)$.

Let $A_{0}(t)$ be the operator defined by

$$
A_{0}(t) u=\sum a_{j}(x, t) D_{j} u+b(x, t) u,
$$

with domain, $D\left(A_{0}(t)\right)$, equal to $H_{P_{t}}^{1}(\Omega)$. Here $P_{t}(x) \equiv P(x, t)$ and $D_{j} \equiv \partial / \partial x_{j}$. Let $A(t)$ denote the closure of $A_{0}(t)$ regarded as an unbounded operator in $X$. Friedrichs [5] and Lax and Phillips [10] have shown that $A(t)+\beta_{t}$ is $m$-accretive if

$$
\beta_{t}=\sup \left\{\left|b^{\prime}(x, t)\right|: x \in \Omega\right\},
$$


where $b^{\prime}=\frac{1}{2}\left(b+{ }^{t} b-\sum \partial a_{j} / \partial x_{j}\right)$. (Recall that an operator $A$ in a real Hilbert space $X$ is accretice if $(A u, u) \geqq 0$ for all $u \in D(A)$. $A$ is $m$-accretive if $A+\lambda$ has range $X$ for all $\lambda>0$. This implies $-A$ generates a $C_{0}$-semigroup of contractions in $X$.) Thus $-A(t)$ generates a $C_{0}$-semigroup in $X$.

The following are the main results.

THEOREM 1. There exists an isomorphism $S(t)$ from $H_{P_{t}}^{1}(\Omega)$ onto $X$ such that

$$
S(t) A(t) S(t)^{-1}=A(t)+B(t),
$$

where $B(t)$ is a bounded operator on $X$.

Remark. According to Proposition 2.4 of [9], Theorem 1 implies that the subspace $H_{P_{t}}^{1}(\Omega)$ is admissible with respect to $A(t)$. This means (see [9, Definition 2.1]) the semigroup generated by $-A(t)$ leaves $H_{P_{t}}^{1}(\Omega)$ invariant and forms a $C_{0}$-semigroup in this space.

THEOREM 2. If $P(x, t)=P(x)$ does not vary with $t$, then $S(t)$ in Theorem 1 may be chosen so that it is continuously differentiable on $[0, T]$ to $B\left(H_{P}^{1}(\Omega), X\right)$ and $B(t)$ is continuous on $[0, T]$ to $B(X)$.

Remark. Suppose that $P(x, t)=P(x)$ does not vary with $t$ so that Theorems 1 and 2 are true. Then Theorems 4.1 and 6.1 of [9] may be applied to the family $\{A(t)\}$, taking $H_{P}^{1}(\Omega)$ for the subspace $Y$ in those two theorems. Note that the stability condition (i) of Theorem 4.1 is true with $M=1$ and $\beta=\sup _{t}\left\{\beta_{t}\right\}$, since the operators $A(t)+\beta_{t}$ are $m$-accretive. The condition (iii) of Theorem 4.1 is also easily seen to be true.

We then have the following result for equation (1.1).

THEOREM 3. Suppose $\phi \in H_{P_{0}}^{1}(\Omega)$ and the map $t \rightarrow f(\cdot, t)$ is continuous on $[0, T]$ to $H^{1}(\Omega)$ so that $f(\cdot, t)$ belongs to $H_{P_{t}}^{1}(\Omega)$ for $0 \leqq t \leqq T$. Then (1.1) has a unique solution $u(x, t)$ such that the map $t \rightarrow u(\cdot, t)$ is continuously differentiable on $[0, T]$ to $X$ and $u(\cdot, t)$ belongs to $H_{P_{t}}^{1}(\Omega)$ for $0 \leqq t \leqq T$.

REMARK. If $P(x, t)=P(x)$ is independent of $t$, then the conclusions of Theorem 3 follow directly from Theorem 7.1 of [9]. We shall show that the general case where $P(x, t)$ varies with $t$ may be reduced to the case $P(x, t)=P(x, 0)$ by an orthogonal transformation of the dependent variables.

The remainder of the paper is devoted to proving the above results. In $\$ 2$ we construct the operator $S(t)$. $\$ 3$ contains inequalities involving commutators. These are used in $\S 4$ to show that $S(t)$ is an isomorphism from $H_{P_{t}}^{1}(\Omega)$ onto $X$ and to establish a regularity result for $S(t)$. Theorems 1,2 , and 3 are proved in $\S 5$.

The author wishes to thank Professor T. Kato, who suggested this problem, for his assistance and guidance in this research.

2. Construction of the operator $S$. We first consider Theorem 1. Here the variable $t$ is only a parameter, and we shall omit it in the discussion and simply write $a_{j}(x), S, \ldots$, for $a_{j}(x, t), S(t)$, etc. 
By the Stone-Weierstrass Theorem, the set of all real-valued functions of class $C^{3}$ on $\Gamma$ is dense in the Banach space of real-valued, continuous functions on $\Gamma$. It follows that, given any $\varepsilon>0$, there exists a $C^{3}$ vector field $\nu$ such that $|-n(y)-\nu(y)| \leqq \varepsilon$ for all $y \in \Gamma$, where $n$ is the exterior unit normal to $\Gamma$. We choose such a $\nu$ so that this inequality is satisfied for some $\varepsilon<1$. Then $\nu(y)$ points into the interior of $\Omega$ for each $y \in \Gamma$, i.e. $(\nu(y), n(y))<0$.

Using this $\nu$, we introduce new coordinates near $\Gamma$ as follows. Let $\omega=\Gamma \times[0, \sigma]$, where $\sigma$ is chosen small enough so that certain conditions stated below are satisfied. Consider the mapping $\omega \rightarrow R^{m}$ defined by

$$
(y, s) \rightarrow y+s \nu(y), \quad(y, s) \in \omega .
$$

Using the fact that $\nu(y)$ is nowhere tangential to $\Gamma$, it follows that the derivative of (2.1) is nonsingular for $s=0$. Using the inverse function theorem, one may then show that (2.1) is a diffeomorphism if $\sigma$ is chosen sufficiently small. Denoting the range of $(2.1)$ by $\Omega^{\prime}$ (note that $\Omega^{\prime} \subset \bar{\Omega}$ ), the inverse $\Omega^{\prime} \rightarrow \omega$ has the form

$$
x \rightarrow(y(x), s(x)), \quad x \in \Omega^{\prime} .
$$

Thus, $y(x) \in \Gamma$ and $s(x) \in[0, \sigma]$ may be thought of as new coordinates for $x \in \Omega^{\prime}$. The matrix

$$
c(x)=\sum a_{j}(x)\left(\partial s / \partial x_{j}\right), \quad x \in \Omega^{\prime},
$$

has the property that it is a strictly negative scalar multiple of the boundary matrix $a_{n}(x)$ for $x \in \Gamma$. This is because the vector $\left(\partial s / \partial x_{1}, \ldots, \partial s / \partial x_{m}\right)$ is an interior normal to $\Omega$, since $\Gamma=\left\{x \in \Omega^{\prime}: s(x)=0\right\}$. Since $a_{n}$ is nonsingular, $\sigma$ may be chosen so that $c(x)$ is nonsingular for $x \in \Omega^{\prime}$.

The spaces $L^{2}\left(\Omega^{\prime}\right)$ and $H^{k}\left(\Omega^{\prime}\right)$ are defined in the same way as $L^{2}(\Omega)$ and $H^{k}(\Omega)$. $L^{2}(\omega)$ denotes the space of vector-valued functions on $\omega=\Gamma \times[0, \sigma]$ which are square integrable with respect to the product measure on $\omega$. We consider $\omega$ as a compact, $C^{3}$ manifold-with-boundary, and we shall use the Sobolev spaces $H^{k}(\omega)$, $-3 \leqq k \leqq 3$. For the definition and basic properties of these spaces, see Hörmander [6].

Let the operators $U_{0}, U: L^{2}\left(\Omega^{\prime}\right) \rightarrow L^{2}(\omega)$ be defined by

$$
\begin{aligned}
U_{0} u(y, s) & =u(y+s v(y)), \quad(y, s) \in \omega, \\
U u & =U_{0} h u,
\end{aligned}
$$

for $u \in L^{2}\left(\Omega^{\prime}\right)$. Here $h(x)=|j(x)|^{-1 / 2}$, where $j(x)$ is the Jacobian of the mapping (2.2). Since the map (2.1) is of class $C^{3}, U_{0}$ is an isomorphism from $H^{k}\left(\Omega^{\prime}\right)$ onto $H^{k}(\omega)$ for $0 \leqq k \leqq 3$. However $h$ is only of class $C^{2}$, so $U$ is an isomorphism between $H^{k}\left(\Omega^{\prime}\right)$ (resp. $\left.H_{0}^{k}\left(\Omega^{\prime}\right)\right)$ and $H^{k}(\omega)$ (resp. $\left.H_{0}^{k}(\omega)\right)$ only for $0 \leqq k \leqq 2$. Using the change of variables formula for integrals, one sees that $U$ is unitary from $L^{2}\left(\Omega^{\prime}\right)$ to $L^{2}(\omega)$. By duality, $U$ extends to an isomorphism between $H^{k}\left(\Omega^{\prime}\right)$ and $H^{k}(\omega)$ for $k=-1$, -2 . 
Let $\phi^{4}+\psi^{4}=1$ be a $C^{3}$ partition of unity for $R^{m}$ with the following properties:

(i) $\phi=1$ and $\psi=0$ in a neighborhood of $\Omega \sim \Omega^{\prime}$.

(ii) The support of $\phi$ is compact in $\Omega$, so $\phi=0$ and $\psi=1$ near $\Gamma$.

(iii) For $x \in \Omega^{\prime}, \phi(x)$ and $\psi(x)$ depend only on $s(x)$. The reason for this last assumption will be discussed in a moment.

In the definition of $S$ we shall use certain matrix-valued functions defined on $\Gamma$ which locally transform the boundary subspace $P(y)$ into a subspace which does not vary with $y$. To construct these functions, we shall use the following lemma.

Lemma 1. Given $y_{0} \in \Gamma$, there exists a neighborhood $\mathscr{U}$ of $y_{0}$ (with respect to $\Gamma$ ) and an orthogonal matrix-valued function $r \in C^{2}(\Gamma)$ such that, for $y \in \mathscr{U}, r(y)$ maps $P(y)$ onto $P=\left\{u \in R^{N}: u_{1}=\cdots=u_{p}=0\right\}$, where $p$ is the common codimension of $P(y)$ for $y$ belonging to the connected component of $\Gamma$ which contains $y_{0}$.

Proof. Clearly there exists $\mathscr{U}$ and $r$ of class $C^{2}$ on $\mathscr{Z}$ with the property that $r(y) P(y)=P$ for $y \in \mathscr{U}$. The problem is to extend $r$ to all of $\Gamma$. This can be done by modifying $r$ near $\partial \mathscr{U}$ so that it is equal to $r\left(y_{0}\right)$ there. For example, by shrinking the neighborhood $\mathscr{U}$, if necessary, and introducing local coordinates, we may assume that we are working in $R^{m-1}, y_{0}=0$, and $\mathscr{U}$ is the ball about $y_{0}$ of radius 1 . We choose a $C^{\infty}$ real-valued function $\rho$ on $[0,1]$ with the property that $\rho(t)=1$ for $t$ near 0 and $\rho(t)=0$ for $t$ near 1 . Then $r^{\prime}(y) \equiv r(\rho(|y|) y)$ has the desired properties.

Using this lemma, we can find an open covering $\mathscr{U}_{1}, \ldots, \mathscr{U}_{K}$ of $\Gamma$ together with orthogonal matrix-valued functions $r_{1}, \ldots, r_{K} \in C^{2}(\Gamma)$ such that, for $k=1, \ldots, K$, and $y \in \mathscr{U}_{k}, r_{k}(y)$ maps $P(y)$ onto

$$
P_{k}=\left\{u \in R^{N}: u_{1}=\cdots=u_{p_{k}}=0\right\} .
$$

( $p_{k}$ is the same for those $\mathscr{U}_{k}$ lying in the same component of $\Gamma$.)

We choose a partition of unity for $\Gamma$

$$
\sum_{k=1}^{K} \zeta_{k}^{2}=1
$$

such that $\zeta_{k}$ has support in $\mathscr{U}_{k}$ for each $k$.

Let $\Delta_{\Gamma}$ denote the Laplace-Beltrami operator on $\Gamma$. This is a negative selfadjoint operator in $L^{2}(\Gamma)$ if we choose $D\left(\Delta_{\Gamma}\right)=H^{2}(\Gamma)$. Let $\Lambda_{\Gamma}=\left(1-\Delta_{\Gamma}\right)^{1 / 2}$. Then $\Lambda_{\Gamma}$ is an isomorphism from $H^{k}(\Gamma)$ onto $H^{k-1}(\Gamma)$ for $1 \leqq k \leqq 3$. By duality $\Lambda_{\Gamma}$ extends to an isomorphism from $H^{k}(\Gamma)$ onto $H^{k-1}(\Gamma)$ for $-2 \leqq k \leqq 0$.

We shall frequently use the natural correspondence whereby a function $u(y, s)$ on $\omega=\Gamma \times[0, \sigma]$ is regarded as a function $u(\cdot, s)$ on $[0, \sigma]$ whose values are functions on $\Gamma$. Under this correspondence $L^{2}(\omega)$ is naturally isomorphic to $L^{2}\left([0, \sigma] ; L^{2}(\Gamma)\right)$, the space of square-integrable functions on $[0, \sigma]$ with values in $L^{2}(\Gamma)$. We also have

$$
H^{k}(\omega) \cong H^{0}\left([0, \sigma] ; H^{k}(\Gamma)\right) \cap \cdots \cap H^{k}\left([0, \sigma] ; H^{0}(\Gamma)\right)
$$


for $k=1,2,3$. Using this correspondence $\Lambda_{\Gamma}$ may be regarded as a bounded operator from $H^{k}(\omega)$ to $H^{k-1}(\omega)$ for $1 \leqq k \leqq 3$. $\Lambda_{\Gamma}$ maps $H_{0}^{k}(\omega)$ into $H_{0}^{k-1}(\omega)$, so, by duality, $\Lambda_{\Gamma}$ extends to a bounded operator from $H^{k}(\omega)$ to $H^{k-1}(\omega)$ for $-2 \leqq k$ $\leqq 0$.

Let $M=U^{-1} \Lambda_{\Gamma} U$. Then $M$ is a bounded operator from $H^{k}\left(\Omega^{\prime}\right)$ to $H^{k-1}\left(\Omega^{\prime}\right)$ for $-1 \leqq k \leqq 2$. Since $U$ is unitary, $M$ is symmetric and bounded below by 1 if it is considered as an operator in $L^{2}\left(\Omega^{\prime}\right)$ by restricting its domain to $H^{1}\left(\Omega^{\prime}\right)$. If we regard $\phi$ and $\psi$ as functions on $\Omega^{\prime}$, then it follows from the assumption (iii) above that $M \phi u=\phi M u$ for $u \in H^{-1}\left(\Omega^{\prime}\right)$ and similarly for $\psi$.

Let $\Lambda=(1-\Delta)^{1 / 2}$ where $\Delta$ is the Laplacian in the whole space $R^{m} . \Lambda$ is an isomorphism from $H^{k}\left(R^{m}\right)$ onto $H^{k-1}\left(R^{m}\right)$ for all $k$.

The expressions $u \rightarrow \phi \Lambda \phi u$ and $u \rightarrow \psi \zeta_{k} r_{k}^{-1} M r_{k} \zeta_{k} \psi u$ define bounded operators from $H^{k}(\Omega)$ to $H^{k-1}(\Omega)$ for $-2 \leqq k \leqq 3$ in the first case and for $-1 \leqq k \leqq 2$ in the second. We are regarding multiplication by $\phi$ as a bounded operator from $H^{k}(\Omega)$ to $H^{k}\left(R^{m}\right)$ and also from $H^{k}\left(R^{m}\right)$ to $H^{k}(\Omega)$ since it has compact support in $\Omega$. In the second case $\psi$ vanishes in a neighborhood of $\Omega \sim \Omega^{\prime}$ so that it is a bounded operator from $H^{k}(\Omega)$ to $H^{k}\left(\Omega^{\prime}\right)$ and also from $H^{k}\left(\Omega^{\prime}\right)$ to $H^{k}(\Omega)$.

The operator $S$ is now defined by

$$
S u=\left(A_{0}+\beta\right) u+\phi \Lambda \phi u+\sum \psi \zeta_{k} r_{k}^{-1} M r_{k} \zeta_{k} \psi u,
$$

for $u \in D(S) \equiv H_{P}^{1}(\Omega)$. Here $\beta=\beta_{t}$ has the value given by (1.2). One sees that $S$ is a bounded operator from $H_{P}^{1}(\Omega)$ to $X$. We shall show in $\S \S 4$ and 5 that $S$ fulfills the requirements of Theorem 1 .

3. Inequalities involving commutators. This section contains results which will be used later to prove Theorems 1 and 2 . We begin with the following proposition which is due to T. Kato (unpublished).

Proposition 1. Let $A$ be a strictly positive selfadjoint operator in a Hilbert space $H$, and let $B \in B(H)$ be such that $B$ maps $D(A)$ into itself with $A B A^{-1} \in B(H)$. If

$$
\|[A, B] u\| \leqq C\left\|A^{1 / 2} u\right\|, \quad\left\|A^{-1 / 2}[A, B] u\right\| \leqq C\|u\|,
$$

for $u \in D(A)$, with a constant $C$, then

$$
\begin{array}{rlrl}
\left\|\left[A^{1 / 2}, B\right] u\right\| & \leqq(C / 2)\|u\|, & & u \in D\left(A^{1 / 2}\right), \\
\left\|\left[A^{1 / 4}, B\right] u\right\| \leqq(C / 2) \pi^{-1 / 2}\left\|A^{-1 / 4} u\right\|, & & u \in D\left(A^{1 / 4}\right) .
\end{array}
$$

Here $[A, B]=A B-B A$ denotes the commutator.

Proof. Using interpolation (see Kato [7]), one sees that $B$ maps $D\left(A^{\alpha}\right)$ into itself with $A^{\alpha} B A^{-\alpha} \in B(H)$ for $0 \leqq \alpha \leqq 1$. Thus $\left[A^{1 / 2}, B\right] u$ and $\left[A^{1 / 4}, B\right] u$ are well defined for $u \in D\left(A^{1 / 2}\right)$ and $u \in D\left(A^{1 / 4}\right)$ respectively.

We claim that (3.1) implies

$$
\left\|A^{-1 / 4}[A, B] u\right\| \leqq C\left\|A^{1 / 4} u\right\|, \quad u \in D(A) .
$$


In order to see this, let $T u=A^{-1 / 2}[A, B] u, u \in D(A)$. By (3.1), $T$ and $A^{1 / 2} T A^{-1 / 2}$ extend to bounded operators on $H$ with norm bounded by $C$. Using interpolation, we obtain $\left\|A^{1 / 4} T A^{-1 / 4}\right\| \leqq C$, which implies (3.4).

We now consider $\left[A^{1 / 2}, B\right]$. Since $A^{1 / 2} B A^{-1 / 2}$ belongs to $B(H)$ and $D(A)$ is a core of $A^{1 / 2}$, it suffices to prove (3.2) only for $u \in D(A)$. For such $u, A^{1 / 2} u$ is given by

$$
A^{1 / 2} u=\pi^{-1} \int_{0}^{\infty} \lambda^{-1 / 2} R(\lambda) A u d \lambda
$$

where $R(\lambda)=(\lambda+A)^{-1}$ is the resolvent of $-A$. For fractional powers, see Kato [8] and Yosida [14]. Since $[R(\lambda) A, B]=\lambda[B, R(\lambda)]=\lambda R(\lambda)[A, B] R(\lambda)$, it follows that

$$
\left[A^{1 / 2}, B\right] u=\pi^{-1} \int \lambda^{1 / 2} R(\lambda)[A, B] R(\lambda) u d \lambda .
$$

Here and in the following, integrals are from 0 to $\infty$. If $v \in D(A)$, then

$$
\left(\left[A^{1 / 2}, B\right] u, v\right)=\pi^{-1} \int \lambda^{1 / 2}\left(A^{-1 / 4}[A, B] R(\lambda) u, A^{1 / 4} R(\lambda) v\right) d \lambda .
$$

Using (3.4), it follows that

$$
\begin{aligned}
\left|\left(\left[A^{1 / 2}, B\right] u, v\right)\right| \leqq & \pi^{-1} C \int \lambda^{1 / 2}\left\|A^{1 / 4} R(\lambda) u\right\|\left\|A^{1 / 4} R(\lambda) v\right\| d \lambda \\
\leqq & \pi^{-1} C\left\{\int \lambda^{1 / 2}\left(R(\lambda)^{2} A^{1 / 2} u, u\right) d \lambda\right\}^{1 / 2} \\
& \times\left\{\int \lambda^{1 / 2}\left(R(\lambda)^{2} A^{1 / 2} v, v\right) d \lambda\right\}^{1 / 2} .
\end{aligned}
$$

Using the spectral theorem, one has $\int \lambda^{1 / 2} R(\lambda)^{2} d \lambda=(\pi / 2) A^{-1 / 2}$. It follows that $\left|\left(\left[A^{1 / 2}, B\right] u, v\right)\right| \leqq(C / 2)\|u\|\|v\|$, from which (3.2) follows.

The inequality (3.3) is proved in a similar manner. Starting from the formula

$$
A^{1 / 4} u=2^{-1 / 2} \pi^{-1} \int_{0}^{\infty} \lambda^{-3 / 4} R(\lambda) A u d \lambda
$$

one proceeds as before to obtain

$$
\left(\left[A^{1 / 4}, B\right] u, v\right)=2^{-1 / 2} \pi^{-1} \int \lambda^{1 / 4}(R(\lambda) u,[B, A] R(\lambda) v) d \lambda .
$$

Using the first half of (3.1), one obtains

$$
\begin{aligned}
\left|\left(\left[A^{1 / 4}, B\right] u, v\right)\right| & \leqq 2^{-1 / 2} \pi^{-1} C\left\{\int \lambda^{1 / 2}\left(R(\lambda)^{2} u, u\right) d \lambda\right\}^{1 / 2}\left\{\int\left(R(\lambda)^{2} A v, v\right) d \lambda\right\}^{1 / 2} \\
& =(C / 2) \pi^{-1 / 2}\left\|A^{-1 / 4} u\right\|\|v\|
\end{aligned}
$$

from which (3.3) follows. 
Corollary. Let $A$ be as in Proposition 1. Let $T$ be an unbounded operator in $H$ with $D(T)=D\left(A^{1 / 2}\right)$ and $T A^{-1 / 2} \in B(H)$ and such that $T$ maps $D\left(A^{3 / 2}\right)$ into $D(A)$ with $A T A^{-3 / 2} \in B(H)$. If

$$
\|[A, T] v\| \leqq C\|A v\|, \quad\left\|A^{-1 / 2}[A, T] v\right\| \leqq C\left\|A^{1 / 2} v\right\|,
$$

$v \in D\left(A^{3 / 2}\right)$, with a constant $C$, then

$$
\begin{aligned}
& \left\|\left[A^{1 / 2}, T\right] v\right\| \leqq(C / 2)\left\|A^{1 / 2} v\right\|, \quad v \in D(A), \\
& \left\|\left[A^{1 / 4}, T\right] v\right\| \leqq(C / 2) \pi^{-1 / 2}\left\|A^{1 / 4} v\right\|, \quad v \in D\left(A^{3 / 4}\right) .
\end{aligned}
$$

Proof. This follows by applying Proposition 1 to $B=T A^{-1 / 2}$.

The next theorem is a consequence of results from the theory of singular integrals proved by Calderón [1], and it will be used frequently later.

TheOREM (CALDERóN). Let $\Delta$ be the Laplacian on $R^{m}$ and $\Lambda=(1-\Delta)^{1 / 2}$. If $a \in C^{1}\left(R^{m}\right)$, then

$$
\|[\Lambda, a] u\| \leqq C\|a\|_{C^{1}}\|u\|, \quad u \in H^{1}\left(R^{m}\right),
$$

with a constant $C$ independent of $a$ and $u$. If $a \in C^{2}\left(R^{m}\right)$, then

$$
\|[\Lambda, a] u\|_{1} \leqq C^{\prime}\|a\|_{C^{2}}\|u\|_{1}, \quad u \in H^{2}\left(R^{m}\right) .
$$

Here $C^{k}\left(R^{m}\right), k=1,2$, is the class of real $N \times N$ matrix-valued functions on $R^{m}$ which together with their first $k$ derivatives are continuous and bounded on $R^{m}$. \|\|$_{C^{k}}$ is the usual supremum norm in this space. Also, \|\| and \|\|$_{1}$ are the norms in $L^{2}\left(R^{m}\right)$ and $H^{1}\left(R^{m}\right)$, respectively.

The following result is essentially due to Seeley [13], but we give another proof here.

Lemma 2. Let $\Delta_{\Gamma}$ be the Laplace-Beltrami operator on $\Gamma$ and $\Lambda_{\Gamma}=\left(1-\Delta_{\Gamma}\right)^{1 / 2}$. If $a \in C^{2}(\Gamma)$, then

$$
\left\|\left[\Lambda_{\Gamma}, a\right] u\right\|_{k} \leqq C\|a\|_{C^{2}}\|u\|_{k}, \quad u \in H^{k+1}(\Gamma), \quad k=0,1,
$$

with a constant $C$ independent of $a$ and $u$. Here $C^{2}(\Gamma)$ is the Banach space of $C^{2}$ matrix-valued functions on $\Gamma$, and \|\|$_{k}$ denotes the norm in $H^{k}(\Gamma)$.

Proof. For $k=0$ this can be established using Proposition 1 with $H=L^{2}(\Gamma)$, $A=1-\Delta_{\Gamma}$, and $B$ being the operator of multiplication by $a$. The hypothesis (3.1) reduces to showing

$$
\left\|\left[\Delta_{\Gamma}, a\right] u\right\|_{k-1} \leqq \text { const. }\|a\|_{C^{2}}\|u\|_{k}, \quad u \in H^{2}(\Gamma), \quad k=0,1 .
$$

We have

$$
\left[\Delta_{\Gamma}, a\right] u=2(\operatorname{grad}(a), \operatorname{grad}(u))+u \Delta_{\Gamma} a,
$$

and the inequality (3.7) follows from this. Therefore (3.6) is true for $k=0$. 
The case $k=1$ may be proved from the case $k=0$ using the relation $\Lambda_{\Gamma}\left[\Lambda_{\Gamma}, a\right] u$ $=\left[a, \Delta_{\Gamma}\right] u-\left[\Lambda_{\Gamma}, a\right] \Lambda_{\Gamma} u$.

Lemma 3. Let $\Delta_{\Gamma}, \Lambda_{\Gamma}$ be as in Lemma 2. If $a \in C^{2}(\Gamma)$, then

$$
\left\|\left[\Lambda_{\Gamma}^{1 / 2}, a\right] u\right\| \leqq C\|a\|_{C^{2}}\left\|\Lambda_{\Gamma}^{-1 / 2} u\right\|, \quad u \in H^{1}(\Gamma),
$$

with a constant $C$ independent of $a$ and $u$. Here \|\| denotes the norm of $L^{2}(\Gamma)$.

Proof. This is proved using Proposition 1 in the same way that (3.6) was established in the case $k=0$.

The following lemma is again due to Seeley [13], but before stating it we make some notational comments. If $V$ is a vector field on $\Gamma$ and $u$ is a function on $\Gamma$, then $V u=(V, \operatorname{grad}(u))$ is the directional derivative of $u$ in the direction $V$. The set of vector fields of class $C^{k}$ on $\Gamma(k=0,1,2)$ is a Banach space, and a norm for this space may be defined as follows. Let $\left\{U_{i}\right\}$ be a finite covering of $\Gamma$ such that each $U_{i}$ is the domain of a coordinate chart which maps $U_{i}$ onto the unit ball $B \subset R^{m-1}$. With respect to the local coordinates for $U_{i}$ the vector field $V$ can be represented as $\left(V_{i, 1}, \ldots, V_{i, m-1}\right)$, where the $V_{i, j}$ are $C^{k}$ real-valued functions on $B$. We define

$$
\|V\|_{C^{k}}=\sum_{i, j}\left\|V_{i, j}\right\|_{C^{k}(B)}
$$

Lemma 4. Let $\Delta_{\Gamma}, \Lambda_{\Gamma}$ be as in Lemma 2. If $V$ is a $C^{2}$ vector field on $\Gamma$, then

$$
\left\|\left[\Lambda_{\Gamma}, V\right] u\right\|_{k-1} \leqq C\|V\|_{C^{2}}\|u\|_{k}, \quad u \in H^{k+1}(\Gamma), \quad k=1,2,
$$

where the constant $C$ is independent of $V$ and $u$.

Proof. For $k=1$ the inequality (3.8) can be established using the Corollary to Proposition 1 where one takes $H=L^{2}(\Gamma), A=1-\Delta_{\Gamma}$, and $T u=V u$. The hypothesis (3.5) reduces to showing

$$
\left\|\left[\Delta_{\Gamma}, V\right] u\right\|_{k-2} \leqq \text { const. }\|V\|_{C^{2}}\|u\|_{k}, \quad u \in H^{3}(\Gamma), \quad k=1,2 .
$$

This inequality can be proved by showing that it holds in the domain of any coordinate chart. When restricted to such a domain we may assume we are working in $R^{m-1}$. The operators $V$ and $\Delta_{\Gamma}$ become first and second order differential operators respectively, and the inequality (3.9) is shown to be true. Thus (3.8) holds for $k=1$.

The inequality (3.8) for $k=2$ follows from the case $k=1$ together with (3.9) and the relation $\Lambda_{\Gamma}\left[\Lambda_{\Gamma}, V\right] u=\left[V, \Delta_{\Gamma}\right] u-\left[\Lambda_{\Gamma}, V\right] \Lambda_{\Gamma} u$.

Now we obtain formulas for the transformation of the differential operators $D_{j}=\partial / \partial x_{j}$ under the mappings $U, U_{0}$ defined by (2.4). Using these, we consider commutators involving the operator $M$.

Let $(y, s) \rightarrow y+s \nu(y), x \rightarrow(y(x), s(x)), c(x)$, and $h(x)$ be as in $\S 2$. Define $h_{j}=h \partial\left(h^{-1}\right) / \partial x_{j}, y_{j}=\partial y / \partial x_{j}, s_{j}=\partial s / \partial x_{j}$. Put $\alpha_{j}(y, s)=a_{j}(y+s v(y))$ for $(y, s) \in \omega$, 
and let $\gamma, \delta_{j}, \sigma_{j}, V_{j}$ be defined in the same way from $c, h_{j}, s_{j}, y_{j}$, respectively. (Note that $V_{j}$ is a vector field on $\omega$.) Let $\delta=\sum \alpha_{j} \delta_{j}$ and

$$
\mathscr{L} v=\gamma \partial v / \partial s+\sum \alpha_{j} V_{j} v+\delta v, \quad v \in H^{1}(\omega) .
$$

Then we have

LEMMA 5.

$$
\begin{aligned}
U_{0} D_{j} U_{0}^{-1} v & =V_{j} v+\sigma_{j}(\partial v / \partial s), & U D_{j} U^{-1} v & =V_{j} v+\sigma_{j}(\partial v / \partial s)+\delta_{j} v, \\
U \sum a_{j} D_{j} U^{-1} v & =\mathscr{L} v, & \left\|V_{j} v\right\| & \leqq C\left\|\Lambda_{\Gamma} v\right\|,
\end{aligned}
$$

$v \in H^{1}(\omega)$. Here \|\| denotes the norm in $L^{2}(\omega)$.

Proof. In order to show the first relation, note that $U_{0}^{-1} v(x)=v(y(x), s(x))$. Using the chain rule, we have $D_{j} U_{0}^{-1} v=V_{j} v+(\partial v / \partial s)\left(\partial s / \partial x_{j}\right)$, where $V_{j} v$ and $\partial v / \partial s$ are evaluated at $(y, s)=(y(x), s(x))$. The formula for $U_{0} D_{j} U_{0}^{-1}$ then follows. Since $U=U_{0} h$, one may obtain the formula for $U D_{j} U^{-1}$ from the formula for $U_{0} D_{j} U_{0}^{-1}$. Using this and (2.3), one arrives at the third formula.

Note that the vector field $V_{j}$ is tangential to $\Gamma$, i.e. $V_{j}(y, s)$ is a tangent vector to $\Gamma$ for each $(y, s) \in \omega$. Therefore $\left(V_{j} v\right)(s)=V_{j}(s) v(s)$ for $s \in[0, \sigma]$. Here $V_{j}(s)$ denotes the vector field on $\Gamma$ defined by $V_{j}(s)(y)=V_{j}(y, s), y \in \Gamma$. It follows that $\left\|V_{j} v(s)\right\|_{\Gamma}^{2} \leqq C\left\|\Lambda_{\Gamma} v(s)\right\|_{\Gamma}^{2}$, where $C$ is a constant independent of $s$ and \|\|$_{\Gamma}$ is the norm in $L^{2}(\Gamma)$. Integrating from 0 to $\sigma$ gives the last inequality in the lemma.

LEMMA 6. If $a \in C^{2}\left(\Omega^{\prime}\right)$, then

$$
\|[M, a] u\|_{k} \leqq C\|a\|_{C^{2}}\|u\|_{k}, \quad u \in H^{k+1}\left(\Omega^{\prime}\right), \quad k=0,1,
$$

with a constant $C$ independent of $a$ and $u$. Here \|\|$_{k}$ is the norm in $H^{k}\left(\Omega^{\prime}\right)$.

Proof. Under the mapping $U$ this inequality corresponds to the inequality $\left\|\left[\Lambda_{\Gamma}, \alpha\right] v\right\|_{k} \leqq C\|\alpha\|_{C^{2}}\|v\|_{k}$, where $\alpha(y, s)=a(y+s \nu(y)), v=U u$, and the norms are now with respect to $\omega$. This inequality follows from Lemma 2 .

LEMMA 7.

$$
\left\|\left[M, D_{j}\right] u\right\| \leqq C\|u\|_{1}, \quad u \in H^{2}\left(\Omega^{\prime}\right) .
$$

Here \|\| is the norm in $L^{2}\left(\Omega^{\prime}\right)$.

Proof. Using Lemma 5 and transforming to $\omega$ by the mapping $U$, one sees that it suffices to prove

$$
\left\|\left[\Lambda_{\Gamma}, V_{j}+\sigma_{j}(\partial / \partial s)+\delta_{j}\right] v\right\| \leqq \text { const. }\|v\|_{1},
$$

where $v=U u$. This inequality is easily obtained from Lemmas 2 and 4 .

LEMMA 8. If $\rho$ is a $C^{3}$ function with support in the interior of $\Omega^{\prime}$, then

$$
\|[\rho M \rho, \Lambda] u\| \leqq C\|u\|_{1}, \quad\left\|\left[\rho M \rho, \Lambda^{1 / 2}\right] u\right\| \leqq C\left\|\Lambda^{1 / 2} u\right\|,
$$


$u \in H^{2}\left(R^{m}\right)$. Here \|\| and \|\|$_{1}$ denote the norms in $L^{2}\left(R^{m}\right)$ and $H^{1}\left(R^{m}\right)$. We are regarding $u \rightarrow \rho M \rho u$ as a bounded operator from $H^{k}\left(R^{m}\right)$ to $H^{k-1}\left(R^{m}\right)$ for $k=1$, $\frac{3}{2}, 2$.

Proof. Let $M^{\prime}=U_{0}^{-1} \Lambda_{\Gamma} U_{0}=h M h^{-1} . M^{\prime}$ has the advantage that it maps $H^{3}\left(\Omega^{\prime}\right)$ into $H^{2}\left(\Omega^{\prime}\right)$, while $M$ does not. Note that $\left(M-M^{\prime}\right) u=h\left[h^{-1}, M\right] u, u \in H^{1}\left(\Omega^{\prime}\right)$. It follows from Lemma 6 that $M-M^{\prime}$ extends to a bounded operator on $H^{k}\left(\Omega^{\prime}\right)$, $k=0,1$. Thus $\rho M \rho-\rho M^{\prime} \rho$ extends to a bounded operator on $H^{k}\left(R^{m}\right), k=0,1$. Using interpolation, one sees that this is also true for $k=\frac{1}{2}$. Consequently it suffices to prove the inequalities in the lemma with $M$ replaced by $M^{\prime}$.

We can use the Corollary to Proposition 1 with $H=L^{2}\left(R^{m}\right), A=1-\Delta$, and $T u=\rho M^{\prime} \rho u$. The hypothesis reduces to showing

$$
\left\|\left[\Delta, \rho M^{\prime} \rho\right] u\right\|_{k-2} \leqq C\|u\|_{k}, \quad u \in H^{3}\left(R^{m}\right), \quad k=1,2 .
$$

One has

$$
\begin{aligned}
{\left[\Delta, \rho M^{\prime} \rho\right] u } & =\sum\left(D_{j}\left[D_{j}, \rho M^{\prime} \rho\right] u+\left[D_{j}, \rho M^{\prime} \rho\right] D_{j} u\right), \\
{\left[D_{j}, \rho M^{\prime} \rho\right] u } & =\left(\partial \rho / \partial x_{j}\right) M^{\prime} \rho u+\rho\left[D_{j}, M^{\prime}\right] \rho u+\rho M^{\prime}\left(\partial \rho / \partial x_{j}\right) u .
\end{aligned}
$$

Using Lemma 5, we have

$$
\left[D_{j}, M^{\prime}\right] v=U_{0}^{-1}\left[V_{j}, \Lambda_{\Gamma}\right] U_{0} v+U_{0}^{-1}\left[\sigma_{j}, \Lambda_{\Gamma}\right](\partial / \partial s) U_{0} v,
$$

$v \in H^{2}\left(\Omega^{\prime}\right)$. It follows from Lemmas 2 and 4 that

$$
\left\|\left[D_{j}, M^{\prime}\right] v\right\|_{k-1} \leqq C\|v\|_{k}, \quad v \in H^{k+1}\left(\Omega^{\prime}\right), \quad k=1,2 .
$$

Hence

$$
\left\|\left[D_{j}, \rho M^{\prime} \rho\right] u\right\|_{k-1} \leqq C\|u\|_{k}, \quad u \in H^{k+1}\left(R^{m}\right), \quad k=1,2 .
$$

Using duality and the fact that $\left(M^{\prime} u, v\right)=\left(u, h^{-2} M^{\prime} h^{2} v\right), u, v \in H^{1}\left(\Omega^{\prime}\right)$, one can show that this inequality holds for $k=0$. The inequality (3.11) can now be proved by combining this with (3.12).

\section{Properties of the operator $S$.}

Proposition 2. $S$ is an isomorphism from $H_{P}^{1}(\Omega)$ onto $X$.

In the proof of this proposition we shall use the operator $R$ defined by

$$
R u=\left(A_{0}+\beta\right) u+\phi \Lambda \phi u+\psi M \psi u, \quad u \in D(R) \equiv D(S) .
$$

LEMMA 9. $S-1$ and $R-1$ are accretive when considered as operators in $X$, and $S-R$ extends to a bounded operator on $X$. It follows that Proposition 2 is true if and only if $R$ is an isomorphism from $H_{P}^{1}(\Omega)$ onto $X$.

Proof. If we expand $(S u, u)$ using the formula $(2.8)$, then $\left(\left(A_{0}+\beta\right) u, u\right)$ is nonnegative because $A_{0}+\beta$ is accretive, and $(\phi \Lambda \phi u, u)$ is bounded below by $\|\phi u\|^{2}$ 
because $\Lambda$ is selfadjoint and bounded below by 1 when considered as an operator in $L^{2}\left(R^{m}\right)$. We have

$$
\sum\left(M r_{k} \zeta_{k} \psi u, r_{k} \zeta_{k} \psi u\right) \geqq \sum\left(r_{k} \zeta_{k} \psi u, r_{k} \zeta_{k} \psi u\right)=\|\psi u\|^{2}
$$

The inequality on the left is a consequence of the fact that $M$ is symmetric and bounded below by 1 when considered as an operator in $L^{2}\left(\Omega^{\prime}\right)$, and the equality on the right follows from (2.6) and the fact that the $r_{k}$ are orthogonal. Therefore

$$
(S u, u) \geqq\|\phi u\|^{2}+\|\psi u\|^{2} \geqq\|u\|^{2}, \quad u \in D(S) .
$$

Here we have used the fact that $\phi^{2}+\psi^{2} \geqq \phi^{4}+\psi^{4}=1$. The proof that $R-1$ is accretive is the same except the $r_{k}$ and $\zeta_{k}$ do not appear.

One has

$$
(S-R) u=\sum \psi \zeta_{k} r_{k}^{-1}\left[M, r_{k} \zeta_{k}\right] \psi u, \quad u \in D(S)
$$

By Lemma 6, $S-R$ extends to a bounded operator on $X$.

Since $S-1$ and $R-1$ are accretive, it follows that $S$ has range equal to $X$ precisely when $S-1$ is $m$-accretive and similarly for $R$. In general, if two accretive operators differ by a bounded operator, then one is $m$-accretive if the other is. Thus, Proposition 2 is equivalent to the range of $R$ being $X$.

We prove that $R$ has range equal to $X$ by showing (1) $R$ is closed when regarded as an operator in $X$, and (2) its adjoint $R^{*}$ is one-to-one. The first assertion is a consequence of the following lemma.

LEMMA 10. The operator $R$ satisfies

$$
\|u\|_{1} \leqq C\|R u\|, \quad u \in D(R),
$$

with a constant $C$.

Proof. Since $R-1$ is accretive, one has $\|u\| \leqq\|R u\|, u \in D(R)$. Therefore, it suffices to show

$$
\|u\|_{1}^{2} \leqq C\left(\|R u\|^{2}+\|u\|^{2}\right), \quad u \in D(R) .
$$

Here and in the following, $C$ denotes a constant.

Since $\phi^{4}+\psi^{4}=1$, we have

$$
\|u\|_{1}^{2} \leqq C\left(\left\|\phi^{2} u\right\|_{1}^{2}+\left\|\psi^{2} u\right\|_{1}^{2}\right), \quad u \in H^{1}(\Omega) .
$$

Using Calderón's theorem, it is not hard to show

$$
\left\|\phi^{2} u\right\|_{1}^{2} \leqq C\left(\|\phi \Lambda \phi u\|^{2}+\|u\|^{2}\right), \quad u \in H^{1}(\Omega) .
$$

For the term $\psi^{2} u$, we shall establish the following estimate:

$$
\left\|\psi^{2} u\right\|_{1}^{2} \leqq C\left(\|\mathscr{A} u\|^{2}+\|\psi M \psi u\|^{2}+\|u\|^{2}\right), \quad u \in H^{1}(\Omega),
$$


where $\mathscr{A}$ denotes the formal differential operator $\sum a_{j} D_{j}$. In order to show (4.6), we first note that

$$
\|w\|_{1} \leqq C\left(\|\partial w / \partial s\|+\left\|\Lambda_{\Gamma} w\right\|+\|w\|\right), \quad w \in H^{1}(\omega),
$$

where \|\| and \|\|$_{1}$ are the norms in $L^{2}(\omega)$ and $H^{1}(\omega)$. This inequality follows from (2.7) and the fact that $\Lambda_{\Gamma}$ is an isomorphism from $H^{1}(\Gamma)$ onto $L^{2}(\Gamma)$. Now let $\mathscr{L}$ be the operator defined by (3.10). Since $\gamma$ is nonsingular, one has

$$
\|\partial w / \partial s\| \leqq C\left(\|\mathscr{L} w\|+\sum\left\|V_{j} w\right\|+\|w\|\right), \quad w \in H^{1}(\omega) .
$$

If one combines this with the preceding inequality and uses the fact $\left\|V_{j} w\right\|$ $\leqq C\left\|\Lambda_{\Gamma} w\right\|$, proved in Lemma 5 , one obtains

$$
\|w\|_{1} \leqq C\left(\|\mathscr{L} w\|+\left\|\Lambda_{\Gamma} w\right\|+\|w\|\right), \quad w \in H^{1}(\omega) .
$$

Using the formula $U \mathscr{A}=\mathscr{L} U$, proved in Lemma 5 , it follows that

$$
\|v\|_{1} \leqq C(\|\mathscr{A} v\|+\|M v\|+\|v\|), \quad v \in H^{1}\left(\Omega^{\prime}\right),
$$

where the norms are with respect to $\Omega^{\prime}$. Putting $v=\psi^{2} u$ and using the fact that $M$ commutes with multiplication by $\psi$, one obtains (4.6).

Combination of (4.4), (4.5), and (4.6) leads to

$$
\|u\|_{1}^{2} \leqq C\left(\|\mathscr{A} u\|^{2}+\|\phi \Lambda \phi u\|^{2}+\|\psi M \psi u\|^{2}+\|u\|^{2}\right),
$$

$u \in H^{1}(\Omega)$. Keeping this in mind, we expand $\|R u\|^{2}$. Note that in proving (4.3), we may ignore terms in $R$ which act as bounded operators on $X$. Thus we may assume without loss of generality that $b=0, \beta=0$. Then

$$
\|R u\|^{2}=\|\mathscr{A} u\|^{2}+\|\phi \Lambda \phi u\|^{2}+\|\psi M \psi u\|^{2}+2(\mathscr{A} u, K u)+2(\phi \Lambda \phi u, \psi M \psi u),
$$

where $K u=\phi \Lambda \phi u+\psi M \psi u$. In a moment we shall prove

$$
\begin{aligned}
-(\phi \Lambda \phi u, \psi M \psi u) & \leqq C\|u\|_{1}\|u\|, \\
-(\mathscr{A} u, K u) & \leqq C\|u\|_{1}\|u\|,
\end{aligned}
$$

$u \in D(R)$. Combining (4.7), (4.8), and (4.9ab), one arrives at

$$
\|u\|_{1}^{2} \leqq C\left(\|R u\|^{2}+\|u\|_{1}\|u\|\right) .
$$

Since $2\|u\|_{1}\|u\| \leqq C^{-1}\|u\|_{1}^{2}+C\|u\|^{2}$, one obtains (4.3).

We now show (4.9a). Let $\rho$ be a $C^{3}$, real-valued function on $R^{m}$ such that (1) $\rho(x)=\psi(x)$ for $x$ belonging to the support of $\phi$, and (2) the support of $\rho$ is contained in the interior of $\Omega^{\prime}$. Then $\phi \psi=\phi \rho$. It follows that $\phi \psi M \psi u=\rho M \rho \phi u$ since $\phi$ and $M$ commute. Therefore, it suffices to show $-(\Lambda w, \rho M \rho w) \leqq C\|w\|_{1}\|w\|$, where $w=\phi u \in H^{1}\left(R^{m}\right)$ and the inner product and norms are with respect to $R^{m}$. We may assume $w \in H^{2}\left(R^{m}\right)$ since this set is dense in $H^{1}\left(R^{m}\right)$. We have

$$
-(\Lambda w, \rho M \rho w)=-\left(\rho \Lambda^{1 / 2} w, M \rho \Lambda^{1 / 2} w\right)-\left(\left[\rho M \rho, \Lambda^{1 / 2}\right] \Lambda^{1 / 2} w, w\right) .
$$


The first term on the right is nonpositive since $M \geqq 1$. Using Lemma 8 , one sees that the second term is bounded in absolute value by $C\|w\|_{1}\|w\|$.

Finally we show (4.9b). We may assume $u \in H^{2}(\Omega) \cap H_{P}^{1}(\Omega)$ since this set is dense in $H_{P}^{1}(\Omega)$. Since $K$ is symmetric, one has $(\mathscr{A} u, K u)=(\mathscr{A} K u, u)+([K, \mathscr{A}] u, u)$. If one integrates the first term by parts, one obtains $(\mathscr{A} K u, u)=-(K u,(\mathscr{A}+e) u)$ $+\left((K u)_{0}, a_{n} v\right)_{\Gamma}$, where $e=\sum \partial a_{j} / \partial x_{j}$. Here $v=u_{0}$ and $(K u)_{0}$ are the traces of $u$ and $K u$ on $\Gamma$, and $(,)_{\Gamma}$ denotes the inner product in $L^{2}(\Gamma)$. Since $\phi=0$ on $\Gamma$, we have $(K u)_{0}=(\psi M \psi u)_{0}$. Using the fact that $M u=h^{-1} U_{0}^{-1} \Lambda_{\Gamma} U_{0} h u$, one can show $(K u)_{0}$ $=h_{0}^{-1} \Lambda_{\Gamma} h_{0} v$, where $h_{0}$ is the restriction of $h$ to $\Gamma$. It follows that

$$
2(\mathscr{A} u, K u)=([K, \mathscr{A}] u, u)-(K u, e u)+\left(h_{0}^{-1} \Lambda_{\Gamma} h_{0} v, a_{n} v\right)_{\Gamma} .
$$

Using Calderón's theorem and Lemmas 6 and 7, one can show

$$
\|[K, \mathscr{A}] w\| \leqq C\|w\|_{1} \sum\left\|a_{j}\right\|_{C^{2}}, \quad w \in H^{2}(\Omega) .
$$

Therefore, in order to finish the proof of $(4.9 b)$, it suffices to show

$$
-\left(h_{0}^{-1} \Lambda_{\Gamma} h_{0} v, a_{n} v\right)_{\Gamma} \leqq C\|u\|_{1}\|u\| .
$$

Let $\pi(y), y \in \Gamma$, be the orthogonal projection of $R^{N}$ onto $P(y)$. Then $\pi(y) v(y)$ $=v(y)$ since $u \in D(R)$. Thus

$$
\left(h_{0}^{-1} \Lambda_{\Gamma} h_{0} v, a_{n} v\right)_{\Gamma}=\left(\Lambda_{\Gamma} v, \alpha v\right)_{\Gamma}+\left(h_{0}^{-1}\left[\Lambda_{\Gamma}, h_{0} \pi\right] v, a_{n} v\right)_{\Gamma},
$$

where $\alpha=\pi a_{n} \pi$. Note that $\alpha(y) \geqq 0$ for $y \in \Gamma$. By Lemma 2, the second term on the right is bounded in absolute value by $C\|v\|_{\Gamma}^{2}$, where \|\|$_{\Gamma}$ denotes the norm in $L^{2}(\Gamma)$. For the first term on the right we have

$$
\left(\Lambda_{\Gamma} v, \alpha v\right)_{\Gamma}=\left(\Lambda_{\Gamma}^{1 / 2} v, \alpha \Lambda_{\Gamma}^{1 / 2} v\right)_{\Gamma}+\left(\left[\alpha, \Lambda_{\Gamma}^{1 / 2}\right] \Lambda_{\Gamma}^{1 / 2} v, v\right)_{\Gamma}
$$

The first term on the right is nonnegative since $\alpha \geqq 0$, and the second term is bounded by $C\|v\|_{\Gamma}^{2}$, by Lemma 3 . This proves $-\left(h_{0}^{-1} \Lambda_{\Gamma} h_{0} v, a_{n} v\right)_{\Gamma} \leqq C\|v\|_{\Gamma}^{2}$. In order to complete the proof of (4.11), it suffices to show $\|v\|_{\Gamma}^{2} \leqq C\|u\|_{1}\|u\|$. Using a partition of unity and change of variables, this inequality can be reduced to the case where $\Omega=\left\{x \in R^{m}: x_{m}>0\right\}$ and $\Gamma=\left\{x \in R^{m}: x_{m}=0\right\}$. Using integration by parts, we have $(v, v)_{\Gamma}=-2\left(D_{m} u, u\right)$, and the desired inequality follows from this.

In order to finish the proof that the range of $R$ is $X$, it remains to show that $R^{*}$ is one-to-one, where $R^{*}$ is the adjoint of $R$ regarded as an operator in $X$.

The formal adjoint of $A_{0}$ is given by

$$
B_{0} v=-\sum D_{j} a_{j} v+{ }^{t} b v, \quad v \in D\left(B_{0}\right) \equiv H_{Q}^{1}(\Omega),
$$

where $Q(x)=\left(a_{n}(x) P(x)\right)^{\perp}, x \in \Gamma$, is the boundary subspace formally adjoint to $P$. The formal adjoint of $R$ is then defined by

$$
T v=\left(B_{0}+\beta\right) v+\phi \Lambda \phi v+\psi M \psi v, \quad v \in D(T) \equiv H_{Q}^{1}(\Omega) .
$$


$R$ and $T$ are formally adjoint to each other, i.e. $(R u, v)=(u, T v), u \in D(R)$, $v \in D(T)$. It follows that $T$ is closable when regarded as an operator in $X$ and $\tilde{T} \subseteq R^{*}$, where $\tilde{T}$ is the closure of $T$.

The operator $B_{0}+\beta$ is accretive; this is proved by Friedrichs [5]. Using the argument in Lemma 9, one sees that $T-1$ is accretive. Hence $\|u\| \leqq\|\tilde{T} u\|, u \in D(\tilde{T})$, and $\tilde{T}$ is one-to-one. In order to show $R^{*}$ is one-to-one (and to complete the proof of Proposition 2), it suffices to prove the following lemma.

LEMMA 11. $\tilde{T}=R^{*}$.

Proof. In the terminology of Friedrichs [3], $\tilde{T}$ is the strong extension of $T$ and $R^{*}$ is the weak extension of $T$, so the proposition asserts the equivalence of the weak and strong extensions of $T$. Friedrichs ([3], [4], [5]) and Lax and Phillips [10] have shown the identity of weak and strong extensions of first order partial differential operators, and this proof is an extension of their methods to the case at hand.

Let $\xi^{2}+\eta^{2}=1$ be a $C^{3}$ partition of unity for $R^{m}$ with the following properties:

(i) $\xi=1$ and $\eta=0$ in a neighborhood of the support of $\phi$, so that $\xi \phi=\phi$, $\eta \phi=0$, and $\eta \psi=\eta$.

(ii) The support of $\xi$ is relatively compact in $\Omega$; in particular, $\xi=0$ and $\eta=1$ near $\Gamma$.

(iii) For $x \in \Omega^{\prime}, \xi(x)$ and $\eta(x)$ depend only on $s(x)$. This implies that $M \xi u=\xi M u$, $u \in H^{-1}\left(\Omega^{\prime}\right)$, and similarly for $\eta$.

Since the inclusion $\tilde{T} \subseteq R^{*}$ has already been shown, it remains to prove the opposite inclusion. Let $v \in D\left(R^{*}\right)$ with $R^{*} v=f$. We shall show $\xi^{2} v, \eta^{2} v \in D(\tilde{T})$.

We first show $\xi^{2} v \in D(\tilde{T})$. Let $J_{\varepsilon}=(1+\varepsilon \Lambda)^{-1}, \varepsilon>0$. For each $k, J_{\varepsilon}$ maps $H^{k}\left(R^{m}\right)$ into itself with norm uniformly bounded in $\varepsilon$. If $u \in H^{k}\left(R^{m}\right)$, then $J_{\varepsilon} u \rightarrow u$ in $H^{k}\left(R^{m}\right)$ as $\varepsilon \rightarrow 0$. Furthermore $J_{\varepsilon}$ maps $H^{k}\left(R^{m}\right)$ into $H^{k+1}\left(R^{m}\right)$.

Let $H_{\varepsilon} u=\xi J_{\varepsilon} \xi u$, where we regard multiplication by $\xi$ as an operator from $H^{k}(\Omega)$ to $H^{k}\left(R^{m}\right)$ and also from $H^{k}\left(R^{m}\right)$ to $H^{k}(\Omega),|k| \leqq 3$. Then, for $|k| \leqq 3, H_{\varepsilon}$ maps $H^{k}(\Omega)$ into itself with norm uniformly bounded in $\varepsilon$. If $u \in H^{k}(\Omega)$, then $H_{\varepsilon} u \rightarrow \xi^{2} u$ in $H^{k}(\Omega)$. (From now on we omit " $\varepsilon \rightarrow 0$ " when it is clear from the context.) For $-3 \leqq k \leqq 2, H_{\varepsilon}$ maps $H^{k}(\Omega)$ into $H^{k+1}(\Omega)$.

Let $v_{\varepsilon}=H_{\varepsilon} v$. Then $v_{\varepsilon} \in D(T)$ since $\xi=0$ near $\Gamma$. Also $v_{\varepsilon} \rightarrow \xi^{2} v$ in $X$. In order to show $\xi^{2} v \in D(\tilde{T})$, it remains to show $T v_{\varepsilon}$ converges in $X$.

Let $T_{1}: X \rightarrow H^{-1}(\Omega)$ be defined by

$$
T_{1} u=-\sum D_{j} a_{j} u+\left({ }^{t} b+\beta\right) u+\phi \Lambda \phi u+\psi M \psi u, \quad u \in X .
$$

Note that $T_{1}$ is an extension of $T$. It is not hard to see that $T_{1} v=f$. Then we have $T v_{\varepsilon}=H_{\varepsilon} f+\left[T_{1}, H_{\varepsilon}\right] v$. Since $H_{\varepsilon} f \rightarrow \xi^{2} f$ in $X$, it remains to show $\left[T_{1}, H_{\varepsilon}\right] v$ converges in $X$. In fact we shall show

$$
\left[T_{1}, H_{\varepsilon}\right] u \text { converges in } X \text { for all } u \in X \text {. }
$$


The first step is to show

$$
\left\|\left[T_{1}, H_{\varepsilon}\right] u\right\| \leqq C\|u\|, \quad u \in X, \quad \varepsilon>0,
$$

with a constant $C$ independent of $\varepsilon$.

Let $\chi$ be a $C^{\infty}$ real-valued function with compact support in $\Omega$ with the property that $\chi(x)=1$ for $x$ belonging to the support of $\xi$. Then $\chi \xi=\xi$ and

$$
\left[T_{1}, H_{\varepsilon}\right] u=\left[T_{1}, \xi\right] \chi J_{\varepsilon} \xi u+\xi\left[\chi T_{1} \chi, J_{\varepsilon}\right] \xi u+\xi J_{\varepsilon}\left[T_{1}, \xi\right] u .
$$

In the first and third terms on the right we have

$$
\left\|\left[T_{1}, \xi\right] w\right\| \leqq C\|w\|, \quad w \in H^{1}(\Omega) .
$$

This follows from Calderón's theorem and Lemma 6. In the second term we have

$$
\left[\chi T_{1} \chi, J_{\varepsilon}\right] w=\varepsilon_{\varepsilon}\left[\Lambda, \chi T_{1} \chi\right] J_{\varepsilon} w=J_{\varepsilon}\left[\Lambda, \chi T_{1} \chi\right] \Lambda^{-1}\left(1-J_{\varepsilon}\right) w,
$$

$w \in L^{2}\left(R^{m}\right)$. It follows from Calderón's theorem and Lemma 8 that $\left\|\left[\Lambda, \chi T_{1} \chi\right] v\right\|$ $\leqq C\|v\|_{1}, v \in H^{2}\left(R^{m}\right)$. Therefore

$$
\left\|\left[\chi T_{1} \chi, J_{\varepsilon}\right] w\right\| \leqq C\|w\|, \quad w \in H^{1}\left(R^{m}\right), \quad \varepsilon>0 .
$$

Since $H^{1}\left(R^{m}\right)$ is dense in $L^{2}\left(R^{m}\right)$, this holds for $u \in L^{2}\left(R^{m}\right)$. Combining this inequality and (4.15) with (4.14) proves (4.13).

Having established the inequality (4.13), we can prove (4.12) using the following well-known principle:

(4.16) Let $A_{\varepsilon}: X_{1} \rightarrow X_{2}, \varepsilon>0$, be a family of continuous linear operators between Banach spaces $X_{1}$ and $X_{2}$. Suppose the $A_{\varepsilon}$ are uniformly bounded in norm with respect to $\varepsilon$ and $A_{\varepsilon} u$ converges in $X_{2}$ as $\varepsilon \rightarrow 0$ for all $u$ belonging to a dense subset of $X_{1}$. Then $A_{\varepsilon} u$ converges in $X_{2}$ for all $u$ in $X_{1}$.

In the particular case of (4.12) one sees that $\left[T_{1}, H_{\varepsilon}\right] u$ converges in $X$ for all $u \in H^{1}(\Omega)$. Since $H^{1}(\Omega)$ is dense in $X$, the assertion (4.12) follows. This completes the proof that $\xi^{2} v \in D(\tilde{T})$.

In order to show $\eta^{2} v \in D(\tilde{T})$, we transform from $\Omega$ to $\omega$. However, we first make the following observation. Suppose $C$ is a bounded operator on $X$. Then Lemma 11 is true if and only if $(R+C)^{*}$ coincides with the closure of $T+C^{*}$. Thus we may assume without loss of generality that $b=0, \beta=0$ in $R$ and $T$.

Let

$$
E u=\mathscr{L} u+\Lambda_{\Gamma} u,
$$

where the domain of $E$ consists of those $u \in H^{1}(\omega)$ which satisfy the boundary conditions $u(y, 0) \in P(y), u(y, \sigma)=0$ for a.a. $y \in \Gamma$. If $u \in D(E)$, then $\eta U^{-1} u \in D(R)$ and

$$
R \eta U^{-1} u=\eta U^{-1} E u+\sum a_{j}\left(\partial \eta / \partial x_{j}\right) U^{-1} u
$$


Here we have used Lemma 5 and the fact that $\eta \phi=0$. It follows that if $E^{*}$ is the adjoint of $E$ regarded as an operator in $L^{2}(\omega)$, then $U \eta v \in D\left(E^{*}\right)$ with

$$
E^{*} U \eta v=f^{\prime} \equiv U\left(\eta f-\sum\left(\partial \eta / \partial x_{j}\right) a_{j} v\right)
$$

The formal adjoint of $E$ is given by $F w=\mathscr{M} w+\Lambda_{\Gamma} w$, where

$$
\mathscr{M} w=-(\partial / \partial s) \gamma w-\sum V_{j} \alpha_{j} w+\delta_{1} w,
$$

with $\delta_{1}=\delta-\sum \operatorname{div}\left(V_{j}\right) \alpha_{j}$. Here we are using the fact that if $V$ is a vector field on $\Gamma$, then $V$ and $-V-\operatorname{div}(V)$ are formally adjoint as operators in $L^{2}(\Gamma)$. The domain of $F$ consists of those $w \in H^{1}(\omega)$ which satisfy the boundary conditions $w(y, 0)$ $\in Q(y)$ for a.a. $y \in \Gamma$. We note that $(E u, w)=(u, F w), u \in D(E), w \in D(F)$. Thus $\tilde{F} \subseteq E^{*}$. In a moment we shall show $E^{*}=\tilde{F}$. Assuming this, it follows that there exists a sequence $\left\{w_{n}\right\} \subset D(\tilde{F})$ with $w_{n} \rightarrow U \eta v$ and $F w_{n} \rightarrow f^{\prime}$ in $L^{2}(\omega)$. Then $\eta U^{-1} w_{n} \in D(T), \eta U^{-1} w_{n} \rightarrow \eta^{2} v$ in $X$. Using the fact that $U\left(-\sum D_{j} a_{j}\right) U^{-1}=\mathscr{M}$, one can show

$$
T \eta U^{-1} w_{n}=\eta U^{-1} F w_{n}-\sum\left(\partial \eta / \partial x_{j}\right) a_{j} U^{-1} w_{n} .
$$

Thus $T \eta U^{-1} w_{n}$ converges in $X$. We conclude $\eta^{2} v \in D(\tilde{T})$.

It remains to show $E^{*}=\tilde{F}$. We first reduce the problem to the case where the boundary subspace $P(y)$ is independent of $y$. Let $r_{k}, \zeta_{k}$ be as in $\S 2$. Let $v \in D\left(E^{*}\right)$. In order to show $v \in D(\tilde{F})$, it suffices to show $\zeta_{k}^{2} v \in D(\tilde{F}), k=1, \ldots, K$. Fix $k$ and let $\zeta=\zeta_{k}, r=r_{k}$ and

$$
L u=\gamma r^{-1} \partial u / \partial s+\sum \alpha_{j} r^{-1} V_{j} u+\delta r^{-1} u+\Lambda_{\Gamma} r^{-1} u,
$$

with the domain of $L$ consisting of those $u \in H^{1}(\omega)$ which satisfy the boundary conditions

$$
u(y, 0) \in P_{k}, \quad u(y, \sigma)=0, \quad \text { for a.a. } y \in \Gamma .
$$

If $u \in D(L)$, then $\zeta r^{-1} u \in D(E)$ and

$$
E \zeta r^{-1} u=\zeta L u+\sum \alpha_{j} V_{j}\left(\zeta r^{-1}\right) u+\left[\Lambda_{\Gamma}, \zeta\right] r^{-1} u .
$$

By Lemma 2, $\left[\Lambda_{\Gamma}, \zeta\right]$ extends to a bounded operator on $L^{2}(\omega)$. It follows that $\zeta v \in D\left(L^{*}\right)$ with

$$
L^{*} \zeta v=\zeta r E^{*} v-\sum V_{j}(\zeta r) \alpha_{j} v-r\left[\Lambda_{\Gamma}, \zeta\right] v .
$$

The formal adjoint of $L$ is

$$
M w=-(\partial / \partial s) r \gamma w-\sum V_{j} r \alpha_{j} w+r \delta_{1} w+r \Lambda_{\Gamma} w
$$

where the domain of $M$ consists of those $w \in H^{1}(\omega)$ which satisfy the adjoint boundary conditions $w(y, 0) \in Q_{k}(y)$, where

$$
Q_{k}(y)=\left(\gamma(y, 0) r(y)^{-1} P_{k}\right)^{\perp} .
$$


We have $(L u, w)=(u, M w), u \in D(L), w \in D(M)$, so $\tilde{M} \subseteq L^{*}$. In a moment we shall show $L^{*}=\tilde{M}$. Assuming this, it follows that there exists a sequence $\left\{w_{n}\right\} \subset D(M)$ with $w_{n} \rightarrow \zeta v, M w_{n} \rightarrow L^{*} \zeta v$ in $L^{2}(\omega)$. For $y$ belonging to the support of $\zeta$, we have $r^{-1}(y) P_{k}=P(y)$. For such $y$, one has $Q_{k}(y)=Q(y)$. Thus $\left\{\zeta w_{n}\right\} \subset D(F)$. Also $\zeta w_{n} \rightarrow \zeta^{2} v$ and

$$
F \zeta w_{n}=\zeta r^{-1} M w_{n}-\sum V_{j}\left(\zeta r^{-1}\right) r \alpha_{j} w_{n}+\left[\Lambda_{\Gamma}, \zeta\right] w_{n},
$$

which converges in $L^{2}(\omega)$ since $\left[\Lambda_{\Gamma}, \zeta\right]$ extends to a bounded operator on $L^{2}(\omega)$. It follows that $\zeta^{2} v \in D(\tilde{F})$.

We now show $L^{*}=\tilde{M}$. By multiplying $L$ by $r \gamma^{-1}$, we may assume $L$ has the form $L u=\partial u / \partial s+G u$, where $G u=\sum a_{j} V_{j} u+b u+c_{1} \Lambda_{\Gamma} c_{2} u$, where $a_{j}=r \gamma^{-1} \alpha_{j} r^{-1}$, $b=r \gamma^{-1} \delta r^{-1}, c_{1}=r \gamma^{-1}$, and $c_{2}=r^{-1}$. Then $M v=-\partial v / \partial s+H v$, where

$$
H v=-\sum V_{j}{ }^{t} a_{j} v+\left({ }^{t} b-\sum \operatorname{div}\left(V_{j}\right)^{t} a_{j}\right) v+{ }^{t} c_{2} \Lambda_{\Gamma}{ }^{t} c_{1} v
$$

and the adjoint boundary conditions become $v(y, 0) \in P_{k}^{\perp}$.

To show $L^{*} \subseteq \tilde{M}$, we use the mollifier $K_{\varepsilon}=\left(1+\varepsilon \Lambda_{\Gamma}\right)^{-1}, \varepsilon>0$. For $-1 \leqq k \leqq 1$, $K_{\varepsilon}$ maps $H^{k}(\Gamma)$ into itself with norm uniformly bounded in $\varepsilon$. For $u \in H^{k}(\Gamma)$, $K_{\varepsilon} u \rightarrow u$ in $H^{k}(\Gamma)$. Furthermore $K_{\varepsilon}$ maps $H^{k}(\Gamma)$ into $H^{k+1}(\Gamma)$ for $k=-1,0$.

Using the natural correspondence (2.7), we shall regard $K_{\varepsilon}$ as mapping $H^{k}(\omega)$ into itself for $-1 \leqq k \leqq 1$. Then, for $u \in H^{k}(\omega), K_{\varepsilon} u \rightarrow u$ in $H^{k}(\omega)$.

Given $v \in D\left(L^{*}\right)$, let $v_{\varepsilon}=K_{\varepsilon} v$. Then $v_{\varepsilon} \rightarrow v$ in $L^{2}(\omega)$. Since $K_{\varepsilon}$ maps $H^{0}(\Gamma)$ into $H^{1}(\Gamma)$, it follows that the first order derivatives of $v_{\varepsilon}$ along directions tangential to $\Gamma$ lie in $L^{2}(\omega)$. In order to show $v_{\varepsilon}$ belongs to $H^{1}(\omega)$, it suffices to show $\partial v_{\varepsilon} / \partial s$ belongs to $L^{2}(\omega)$.

If $L^{*} v=f$, then $-\partial v / \partial s+H v=f$ when we regard $\partial v / \partial s$ and $H v$ as elements of $H^{-1}(\omega)$. Then $\partial v_{\varepsilon} / \partial s=K_{\varepsilon}(\partial / \partial s) v=-K_{\varepsilon} f+K_{\varepsilon} H v$. One has $K_{\varepsilon} H v \in L^{2}(\omega)$, since $K_{\varepsilon}$ maps $H^{-1}(\Gamma)$ and $L^{2}(\Gamma)$ and the operator $H$ only involves differentiation in the $y$ compcnent of a function $v(y, s)$ on $\omega$. Thus $v_{\varepsilon} \in H^{1}(\omega)$.

We claim $v_{\varepsilon}$ satisfies the boundary conditions $v_{\varepsilon}(y, 0) \in P_{k}^{\perp}$. Suppose $u \in D(L)$. Let $u(0),\left(K_{\varepsilon} u\right)(0)$, denote the traces of $u$ and $K_{\varepsilon} u$ on $\Gamma \times\{0\}$. Then $\left(K_{\varepsilon} u\right)(0)$ $=K_{\varepsilon}(u(0))$. It follows that $K_{\varepsilon} u$ satisfies the boundary conditions (4.19) and hence belongs to $D(L)$. We have

$$
\left(L K_{\varepsilon} u, v\right)=\left(K_{\varepsilon} u, f\right)=\left(u, K_{\varepsilon} f\right),
$$

since $L^{*} v=f$. On the other hand, we have $\left(L K_{\varepsilon} u, v\right)=\left(\partial u / \partial s, v_{\varepsilon}\right)+\left(G K_{\varepsilon} u, v\right)$, since $K_{\varepsilon}$ and $\partial / \partial s$ commute. Integrating by parts, one obtains

$$
\left(\partial u / \partial s, v_{\varepsilon}\right)=-\left(u, \partial v_{\varepsilon} / \partial s\right)-\left(u(0), v_{\varepsilon}(0)\right)_{\Gamma} .
$$

We use here the fact that $u(y, \sigma)=0$ for a.a. $y \in \Gamma$. Thus

$$
\left(L K_{\varepsilon} u, v\right)=-\left(u, \partial v_{\varepsilon} / \partial s\right)-\left(u(0), v_{\varepsilon}(0)\right)_{\Gamma}+\left(v, K_{\varepsilon} H v\right)=\left(u, K_{\varepsilon} f\right)-\left(u(0), v_{\varepsilon}(0)\right)_{\Gamma} .
$$


Combining this with (4.20) gives $\left(u(0), v_{\varepsilon}(0)\right)_{\Gamma}=0, u \in D(L)$. Since the values of $u(0)$ can be chosen arbitrarily subject to the restriction (4.19), it follows that $v_{\varepsilon}$ satisfies the boundary conditions $v_{\varepsilon}(y, 0) \in P_{k}^{\perp}$. Therefore $v_{\varepsilon}$ belongs to $D(M)$.

It remains to show $M v_{\varepsilon} \rightarrow f$ in $L^{2}(\omega)$. We have $M v_{\varepsilon}=K_{\varepsilon} f+\left[H, K_{\varepsilon}\right] v$. Since $f \in L^{2}(\omega), K_{\varepsilon} f \rightarrow f$ in $L^{2}(\omega)$. We claim $\left[H, K_{\varepsilon}\right] v \rightarrow 0$ in $L^{2}(\omega)$. In fact, we shall show

$$
\left[H, K_{\varepsilon}\right] w \rightarrow 0 \text { in } L^{2}(\omega) \text { for all } w \in L^{2}(\omega) .
$$

The proof of this is very similar to (4.12). The crucial step is to establish the inequality

$$
\left\|\left[H, K_{\varepsilon}\right] w\right\| \leqq C\|w\|, \quad w \in L^{2}(\omega), \quad \varepsilon>0,
$$

and then we can apply the principle (4.16). We have

$$
\left[H, K_{\varepsilon}\right] w=K_{\varepsilon}\left[\Lambda_{\Gamma}, H\right] \Lambda_{\Gamma}^{-1}\left(1-K_{\varepsilon}\right) w .
$$

Using Lemmas 2 and 4 , one sees that $\left\|\left[\Lambda_{\Gamma}, H\right] \Lambda_{\Gamma}^{-1} v\right\| \leqq C\|v\|, v \in H^{1}(\omega)$. Using this inequality, one easily obtains (4.22).

Since $\left[H, K_{\varepsilon}\right] w \rightarrow 0$ in $L^{2}(\omega)$ for $w \in H^{1}(\omega)$, and $H^{1}(\omega)$ is dense in $L^{2}(\omega)$, one obtains (4.21). Thus $M v_{\varepsilon} \rightarrow f$ and $v \in D(\tilde{M})$.

This concludes the proof of Proposition 2. We now prove a regularity result for $S$.

Proposition 3. If $v \in H^{1}(\Omega)$ then $S^{-1} v \in H^{2}(\Omega)$.

Proof. Let $u=S^{-1} v$. Then $u \in H_{P}^{1}(\Omega), S u \in H^{1}(\Omega)$, and we must show $u \in H^{2}(\Omega)$. $(S-R) u$ is given by $(4.1)$ and, by Lemma $6,\left[M, r_{k} \zeta_{k}\right]$ is a bounded operator on $H^{1}\left(\Omega^{\prime}\right)$. Therefore $(S-R) u$ belongs to $H^{1}(\Omega)$. Since $S u \in H^{1}(\Omega)$, it follows that $R u \in H^{1}(\Omega)$.

Let $\xi^{2}+\eta^{2}=1$ be the partition of unity introduced in the proof of Lemma 11 . We must show $\xi^{2} u, \eta^{2} u \in H^{2}(\Omega)$.

Let $\left\{J_{\varepsilon}: \varepsilon>0\right\}$ be the operators introduced in the proof of Lemma 11 and $G_{\varepsilon} u=\varepsilon^{-1} \xi\left(1-J_{\varepsilon}\right) \xi u$. Note that $\varepsilon^{-1}\left(1-J_{\varepsilon}\right)=\Lambda J_{\varepsilon}$. We claim that in order to show $\xi^{2} u \in H^{2}(\Omega)$, it suffices to show $G_{\varepsilon} u$ converges in $H^{1}(\Omega)$. Suppose the latter is true. Then

$$
G_{\varepsilon} u-\varepsilon^{-1}\left(1-J_{\varepsilon}\right) \xi^{2} u=\varepsilon^{-1}\left[J_{\varepsilon}, \xi\right] \xi u=J_{\varepsilon}[\xi, \Lambda] J_{\varepsilon} \xi u .
$$

By Calderón's theorem, $[\xi, \Lambda]$ is a bounded operator on $H^{1}\left(R^{m}\right)$, so the right side converges in $H^{1}\left(R^{m}\right)$. Therefore $J_{\varepsilon} \Lambda \xi^{2} u$ converges in $H^{1}\left(R^{m}\right)$. So $\Lambda \xi^{2} u \in H^{1}\left(R^{m}\right)$ which implies $\xi^{2} u \in H^{2}(\Omega)$. This proves the claim.

It remains to show $G_{\varepsilon} u$ converges in $H^{1}(\Omega)$. Note that $G_{\varepsilon} u \in D(R)$ since $\xi=0$ near $\Gamma$. By (4.2), we are reduced to proving $R G_{\varepsilon} u$ converges in $X$. We have

$$
R G_{\varepsilon} u=G_{\varepsilon} R u+\left[R, G_{\varepsilon}\right] u .
$$

Since $R u \in H^{1}(\Omega), G_{\varepsilon} R u \rightarrow \xi \Lambda \xi R u$ in $X$. 
It remains to show that $\left[R, G_{\varepsilon}\right] u$ converges in $X$. In fact we shall show that $\left[R, G_{\varepsilon}\right] w$ converges in $X$ for all $w \in H^{1}(\Omega)$. (Note that in proving this we do not use the fact that $w$ satisfies the boundary conditions $w_{0}(y) \in P(y), y \in \Gamma$, so we shall assume that $P(y)=R^{N}$ and $D(R)=H^{1}(\Omega)$.) The proof is similar to the proof of (4.12). We establish the inequality

$$
\left\|\left[R, G_{\varepsilon}\right] w\right\| \leqq C\|w\|_{1}, \quad w \in H^{1}(\Omega), \quad \varepsilon>0,
$$

and then apply the principle (4.16). Let $\chi$ be the function introduced in connection with the proof of (4.13). Then

$$
\left[R, G_{\varepsilon}\right] w=[R, \xi]_{\chi} \Lambda J_{\varepsilon} \xi w+\xi J_{\varepsilon}[\chi R \chi, \Lambda] J_{\varepsilon} \xi w+\xi \Lambda J_{\varepsilon} \chi[R, \xi] w .
$$

Application of Calderón's theorem and Lemma 6 shows that

$$
\|[R, \xi] w\|_{k} \leqq C\|w\|_{k}, \quad w \in H^{k+1}(\Omega), \quad k=0,1 .
$$

Using Calderón's theorem and Lemma 8, one obtains

$$
\|[\chi R \chi, \Lambda] v\| \leqq C\|v\|_{1}, \quad v \in H^{2}\left(R^{m}\right) .
$$

Combination of these last two inequalities with (4.24) proves (4.23).

Note that if $w \in H^{2}(\Omega)$, then $G_{\varepsilon} w \rightarrow \xi \Lambda \xi w$ in $H^{1}(\Omega)$. Thus $\left[R, G_{\varepsilon}\right] w \rightarrow[R, \xi \Lambda \xi] w$ in $X$ for $w \in H^{2}(\Omega)$. Since $H^{2}(\Omega)$ is dense in $H^{1}(\Omega)$, it follows that $\left[R, G_{\varepsilon}\right] w$ converges in $X$ for all $w \in H^{1}(\Omega)$. Thus we have proved $\xi^{2} u \in H^{2}(\Omega)$.

We now show $\eta^{2} u \in H^{2}(\Omega)$. It suffices to show $U \eta^{2} u \in H^{2}(\omega)$ which in turn reduces to showing $\Lambda_{\Gamma} U \eta^{2} u$ and $(\partial / \partial s) U \eta^{2} u$ belong to $H^{1}(\omega)$. To show this, we shall use the operators $\left\{K_{\varepsilon}: \varepsilon>0\right\}$ introduced in the proof of Lemma 11. In order to show $\Lambda_{\Gamma} U \eta^{2} u \in H^{1}(\omega)$, it suffices to show $K_{\varepsilon} \Lambda_{\Gamma} U \eta^{2} u=\varepsilon^{-1}\left(1-K_{\varepsilon}\right) U \eta^{2} u$ converges in $H^{1}(\omega)$. Since $\eta$ commutes with $U^{-1} K_{\varepsilon} U$, it suffices to show $\varepsilon^{-1} \eta U^{-1}\left(1-K_{\varepsilon}\right) w$ converges in $H^{1}(\Omega)$, where $w=U \eta u$.

Let $L_{\varepsilon}=\sum \zeta_{k} r_{k}^{-1} K_{\varepsilon} r_{k} \zeta_{k}$. Using (2.6), we have

$$
\varepsilon^{-1}\left(K_{\varepsilon}-L_{\varepsilon}\right) w=\varepsilon^{-1} \sum \zeta_{k} r_{k}^{-1}\left[r_{k} \zeta_{k}, K_{\varepsilon}\right] w .
$$

Note that $\varepsilon^{-1}\left[r_{k} \zeta_{k}, K_{\varepsilon}\right] w=K_{\varepsilon}\left[\Lambda_{\Gamma}, r_{k} \zeta_{k}\right] K_{\varepsilon} w$. This converges in $H^{1}(\omega)$ as $\varepsilon \rightarrow 0$ by Lemma 2. Thus it suffices to show

$$
\varepsilon^{-1} \eta U^{-1}\left(1-L_{\varepsilon}\right) w \text { converges in } H^{1}(\Omega) .
$$

We claim that $\varepsilon^{-1} \eta U^{-1}\left(1-L_{\varepsilon}\right) w$ belongs to $D(R)$. Since $u \in D(R)$, it follows that $w(y, 0) \in P(y)$ for a.a. $y \in \Gamma$. Therefore $r_{k}(y) \zeta_{k}(y) w(y, 0) \in P_{k}$ for a.a. $y \in \Gamma$, which implies $\left(K_{\varepsilon} r_{k} \zeta_{k} w\right)(y, 0) \in P_{k}$ for a.a. $y \in \Gamma$. From this one concludes $\left(L_{\varepsilon} w\right)(y, 0)$ $\in P(y)$ for a.a. $y \in \Gamma$. The claim then follows.

Using (4.2), one sees that the assertion (4.26) reduces to showing $\varepsilon^{-1} R \eta U^{-1}\left(1-L_{\varepsilon}\right) w$ converges in $X$. We may assume without loss of generality that $b=0, \beta=0$ in $R$. Let $E$ be the operator given by (4.17). Using (4.18), one sees that it suffices to show $\varepsilon^{-1} E\left(1-L_{\varepsilon}\right) w$ converges in $L^{2}(\omega)$. For this we do not use 
the fact that $w$ satisfies the boundary conditions $w(y, 0) \in P(y), y \in \Gamma$, so we shall assume that $P(y)=R^{N}$ and $D(E)=H^{1}(\omega)$. Again using (4.25), one sees that it suffices to show $\varepsilon^{-1} E\left(1-K_{\varepsilon}\right) w$ ' converges in $L^{2}(\omega)$. One has

$$
\varepsilon^{-1} E\left(1-K_{\varepsilon}\right) w=\varepsilon^{-1}\left(1-K_{\varepsilon}\right) E w+\varepsilon^{-1}\left[K_{\varepsilon}, E\right] w .
$$

It is not hard to show that $R u \in H^{1}(\Omega)$ implies $E w \in H^{1}(\omega)$. Therefore the first term on the right converges to $\Lambda_{\Gamma} E w$ in $L^{2}(\omega)$.

It remains to show $\varepsilon^{-1}\left[K_{\varepsilon}, E\right] w$ converges in $L^{2}(\omega)$. We shall show that $\varepsilon^{-1}\left[K_{\varepsilon}, E\right] v$ converges in $X$ for all $v \in H^{1}(\omega)$. We first establish the inequality

$$
\left\|\varepsilon^{-1}\left[K_{\varepsilon}, E\right] v\right\| \leqq C\|v\|_{1}, \quad v \in H^{1}(\omega), \quad \varepsilon>0,
$$

and then apply (4.16). We have

$$
\varepsilon^{-1}\left[K_{\varepsilon}, E\right] v=K_{\varepsilon}\left[E, \Lambda_{\Gamma}\right] K_{\varepsilon} v .
$$

Using Lemmas 2 and 5 , one sees that $\left\|\left[E, \Lambda_{\Gamma}\right] u\right\| \leqq C\|u\|_{1}, u \in H^{2}(\omega)$. Combining this with (4.28), one obtains (4.27). Since $\varepsilon^{-1}\left[K_{\varepsilon}, E\right] v=\varepsilon^{-1}\left[\left(I-K_{\varepsilon}\right), E\right] v$ converges to $\left[\Lambda_{\Gamma}, E\right] v$ in $L^{2}(\omega)$ for $v \in H^{2}(\omega)$ and $H^{2}(\omega)$ is dense in $H^{1}(\omega)$, it follows that $\varepsilon^{-1}\left[K_{\varepsilon}, E\right] v$ converges in $X$ for all $v \in H^{1}(\omega)$. This concludes the proof that $\Lambda_{\Gamma} U \eta^{2} u \in H^{1}(\omega)$.

It remains to show $(\partial / \partial s) U \eta^{2} u \in H^{1}(\omega)$. It was noted above that $E w=E U \eta u$ $\in H^{1}(\omega)$. It follows that $E U \eta^{2} u \in H^{1}(\omega)$. Using the fact that $\Lambda_{\Gamma} U \eta^{2} u \in H^{1}(\omega)$ and the fact that the $V_{j} \Lambda_{\Gamma}^{-1}$ are bounded operators in $H^{1}(\omega)$, it follows that $\gamma(\partial / \partial s) U \eta^{2} u \in H^{1}(\omega)$. Since $\gamma$ is nonsingular, this implies $(\partial / \partial s) U \eta^{2} u \in H^{1}(\omega)$.

5. Proof of Theorems 1-3. In order to show that $S$ defined by (2.8) satisfies the requirements of Theorem 1, it remains to prove the following proposition.

Proposition 4. $S A S^{-1}=A+B$, where $B \in B(X)$.

Proof. We first show that $u \in H_{P}^{1}(\Omega)$ implies $A S^{-1} u \in H_{P}^{1}(\Omega)$. Let $v=S^{-1} u$. Then $S v=u \in H_{P}^{1}(\Omega)$ and, by Proposition 3, $v \in H_{P}^{1}(\Omega) \cap H^{2}(\Omega)$. We must show $A v \in H_{P}^{1}(\Omega)$.

Note that $\phi \Lambda \phi v \in H_{P}^{1}(\Omega)$ since $\phi=0$ near $\Gamma$. Next we claim

$$
\psi \zeta_{k} r_{k}^{-1} M r_{k} \zeta_{k} \psi v \in H_{P}^{1}(\Omega), \quad k=1, \ldots, K .
$$

To prove this, note that $v \in H_{P}^{1}(\Omega) \cap H^{2}(\Omega)$ implies $r_{k} \zeta_{k} \psi v \in H^{2}\left(\Omega^{\prime}\right)$ and $\left(r_{k} \zeta_{k} \psi v\right)(y) \in P_{k}$ for a.a. $y \in \Gamma$ since $r_{k}(y)$ maps $P(y)$ onto $P_{k}$ for $y$ belonging to the support of $\zeta_{k}$. Therefore $w=U r_{k} \zeta_{k} \psi v \in H^{2}(\omega)$ and $w(y, 0) \in P_{k}$ for a.a. $y \in \Gamma$. It follows that $\left(\Lambda_{\Gamma} w\right)(y, 0) \in P_{k}$ for a.a. $y \in \Gamma$ and $\left(M r_{k} \zeta_{k} \psi v\right)(y) \in P_{k}$ for a.a. $y \in \Gamma$. The assertion (5.1) follows from this. We conclude $A v \in H_{P}^{1}(\Omega)$.

It follows that $H_{P}^{1}(\Omega) \subset D\left(S A S^{-1}\right)$, and for $u \in H_{P}^{1}(\Omega)$ we have

$$
S A S^{-1} u-A u=[S, A] S^{-1} u=[Z, A] S^{-1} u,
$$

with

$$
Z v=\phi \Lambda \phi v+\sum \psi \zeta_{k} r_{k}^{-1} M r_{k} \zeta_{k} \psi v, \quad v \in H^{1}(\Omega)
$$


Note that $Z$ is an extension of $S-A-\beta$. We claim

$$
\|[Z, A] v\| \leqq C\|v\|_{1}\left(\sum\left\|a_{j}\right\|_{C^{2}}+\|b\|_{C^{1}}\right), \quad v \in H_{P}^{1}(\Omega) \cap H^{2}(\Omega) .
$$

If $K v=\phi \Lambda \phi v+\psi M \psi v$ is as in Lemma 10 , then $(Z-K) v=(S-R) v$ is given by (4.1) with $u$ replaced by $v$. We have already noted that $S-R$ is a bounded operator on both $X$ and $H^{1}(\Omega)$. Therefore (5.2) follows from (4.10) since $A v=\mathscr{A} v+b v$.

It follows from (5.2) that $B=[Z, A] S^{-1}$ extends to a bounded operator on $X$. Thus we have $S A S^{-1} u=A u+B u$ for $u \in H_{P}^{1}(\Omega)$. Since $H_{P}^{1}(\Omega)$ is a core of $A$, it follows that this holds for $u \in D(A)$. So $S A S^{-1}$ is an extension of $A+B$. It follows that $S(A+\lambda) S^{-1} \supset A+B+\lambda$ for all $\lambda$. If $\lambda$ is large, the right side has range $X$ and the left side is one-to-one. Therefore we have equality: $S A S^{-1}=A+B$.

Theorem 1 is a direct consequence of Propositions 2 and 4.

Proof of Theorem 2. Let

$$
S(t) u=\left(A_{0}(t)+\beta\right) u+\phi \Lambda \phi u+\sum \psi \zeta_{k} r_{k}^{-1} M r_{k} \zeta_{k} \psi u,
$$

for $u \in D(S(t)) \equiv H_{P}^{1}(\Omega)$. Here $\beta=\sup _{t}\left\{\beta_{t}\right\}$, where $\beta_{t}$ has the value (1.2). The matrix

$$
c(x, t)=\sum a_{j}(x, t) \partial s / \partial x_{j}, \quad x \in \Omega^{\prime}, \quad 0 \leqq t \leqq T,
$$

defined by (2.3), is now a function of $t$, as well as $x$. Therefore the value of $\sigma$ in (2.1) should be chosen so that $c(x, t)$ is nonsingular for $x \in \Omega^{\prime}, 0 \leqq t \leqq T$.

Note that the only part of $S(t)$ which varies with $t$ is $A_{0}(t)$. Thus $S(t)$ is continuously differentiable on $[0, T]$ to $B\left(H_{P}^{1}(\Omega), X\right)$, since this is true for $A_{0}(t)$.

According to Propositions 2 and 4, $S(t)$ is an isomorphism from $H_{P}^{1}(\Omega)$ onto $X$, and $S(t) A(t) S(t)^{-1}=A(t)+B(t)$, where $B(t) \in B(X)$. It follows from the inequality (5.2) that the map $t \rightarrow B(t)=[Z, A(t)] S(t)^{-1}$ is continuous on $[0, T]$ to $B(X)$.

Proof of Theorem 3. We noted in the remark after Theorem 3 that the conclusions of Theorem 3 are true if $P(x, t)=P(x)$ is independent of $t$. We turn now to the general case where $P(x, t)$ varies with $t$.

Suppose $r(x, t)$ is an orthogonal matrix-valued function of class $C^{2}$ on $\bar{\Omega} \times[0, T]$ with the property that

$$
r(y, t) \text { maps } P(y, 0) \text { onto } P(y, t) \text { for } y \in \Gamma, \quad 0 \leqq t \leqq T .
$$

If one makes the change of variables

$$
v(x, t)=r(x, t)^{-1} u(x, t), \quad x \in \bar{\Omega}, \quad 0 \leqq t \leqq T,
$$

then equation (1.1) for $u$ corresponds to the following equation for $v$.

$$
\begin{aligned}
& \partial v / \partial t+\sum r^{-1} a_{j} r \partial v / \partial x_{j}+b^{\prime} v=r^{-1} f, \\
& v(x, 0)=r(x, 0)^{-1} \phi(x), \\
& v(x, t) \in P(x, 0), \quad x \in \Gamma, \quad 0 \leqq t \leqq T,
\end{aligned}
$$


where $b^{\prime}=r^{-1}\left(\partial r / \partial t+\sum a_{j} \partial r / \partial x_{j}+b r\right)$. In this equation the boundary subspace $P(x, 0)$ does not vary with $t$. We also note that the assumption $f(\cdot, t) \in H_{P_{t}}^{1}(\Omega)$ implies $r(\cdot, t)^{-1} f(\cdot, t) \in H_{P_{0}}^{1}(\Omega)$. Therefore, as noted above, the conclusions of Theorem 3 hold for this equation. It follows that $u=r v$ is the desired solution to equation (1.1).

To complete the argument, one must show that there is a function $r(x, t)$ with the above properties. The condition (5.3) can be restated as

$$
r(y, t) e(y, 0) r(y, t)^{-1}=e(y, t), \quad y \in \Gamma, \quad 0 \leqq t \leqq T,
$$

where $e(y, t)$ is the orthogonal projection of $R^{N}$ onto $P(y, t)$. Such an $r$ is called a transformation function for $e(y, t)$, and it may be constructed using Kato's method (see [8, p. 99]). We take for $r$ the solution of the differential equation

$$
(\partial / \partial t) r(x, t)=q(x, t) r(x, t), \quad 0 \leqq t \leqq T,
$$

$r(x, 0)=1$, where $q=[\partial e / \partial t, e]$. Since $q$ is antisymmetric, this $r$ is an orthogonal matrix-valued function of class $C^{2}$ on $\Gamma \times[0, T]$ which satisfies (5.4) with $r(y, 0)=1$. We must extend $r$ to $\bar{\Omega} \times[0, T]$. Let $r^{\prime}(y, s, t)=r(y, t \rho(s))$, where $\rho$ is a function mapping $[0, \sigma]$ into $[0,1]$ with $\rho(0)=1$ and $\rho(s)=0$ for $s \geqq \sigma / 2 . r^{\prime}$ is defined on $\Gamma \times[0, \sigma] \times[0, T]$ with $r^{\prime}(y, 0, t)=r(y, t)$ and $r^{\prime}(y, s, t)=1$ for $s \geqq \sigma / 2$. Letting $y(x), s(x)$ be the coordinates in $\Omega^{\prime}$ introduced in (2.2), one may change variables to obtain $r(x, t)=r^{\prime}(y(x), s(x), t)$. This $r$ is defined on $\Omega^{\prime} \times[0, T]$ with $r(x, t)=1$ if $s(x) \geqq \sigma / 2$. $r$ may then be extended to $\bar{\Omega} \times[0, T]$ by setting $r(x, t)=1$ for $x \in \bar{\Omega}$ $\sim \Omega^{\prime}$.

\section{BIBLIOGRAPHY}

1. A. P. Calderón, Commutators of singular integral operators, Proc. Nat. Acad. Sci. U.S.A. 53 (1965), 1092-1099. MR 31 \#1575.

2. H. O. Cordes and R. D. Moyer, On the differentiability of strong solutions of partial differential equations, Comm. Pure Appl. Math. 17 (1964), 435-450. MR 30 \#2221.

3. K. O. Friedrichs, The identity of weak and strong extensions of differential operators, Trans. Amer. Math. Soc. 55 (1944), 132-151. MR 5, 188.

4. - Symmetric hyperbolic linear differential equations, Comm. Pure Appl. Math. 7 (1954), 345-392. MR 16, 44.

5. - Symmetric positive linear differential equations, Comm. Pure Appl. Math. 11 (1958), 333-418. MR 20 \#7147.

6. L. Hörmander, Linear partial differential operators, Die Grundlehren der math. Wissenschaften, Band 116, Academic Press, New York; Springer-Verlag, Berlin, 1963. MR 28 \#4221.

7. T. Kato, A generalization of the Heinz inequality, Proc. Japan Acad. 37 (1961), 305-308. MR 26 \#2876.

8. - Perturbation theory for linear operators, Die Grundlehren der math. Wissenschaften, Band 132, Springer-Verlag, New York, 1966. MR 34 \#3324.

9. - Linear evolution equations of "hyperbolic" type, J. Fac. Sci. Univ. Tokyo 17 (1970), 241-258.

10. P. D. Lax and R. S. Phillips, Local boundary conditions for dissipative symmetric linear differential operators, Comm. Pure Appl. Math. 13 (1960), 427-455. MR 22 \#9718. 
11. J. L. Lions and E. Magenes, Problèmes aux limites non homogènes et applications. Vol. 1, Travaux et Recherches Mathématiques, no. 17, Dunod, Paris, 1968. MR 40 \#512.

12. C. B. Morrey, Jr., Multiple integrals in the calculus of variations, Die Grundlehren der math. Wissenschaften, Band 130, Springer-Verlag, New York, 1966. MR 34 \#2380.

13. R. T. Seeley, Singular integrals on compact manifolds, Amer. J. Math. 81 (1959), 658-690. MR 22 \#905.

14. K. Yosida, Functional analysis, 2nd ed., Die Grundlehren der math. Wissenschaften, Band 123, Springer-Verlag, New York, 1968. MR 39 \#741.

Department of Mathematics, University of California, Berkeley, California 94720

Department of Mathematics, University of Kentucky, Lexington, Kentucky 40506 